\title{
Well-posedness in critical spaces for the compressible Navier-Stokes equations with density dependent viscosities
}

\author{
Qionglei Chen, Changxing Miao and Zhifei Zhang
}

\begin{abstract}
In this paper, we prove the local well-posedness in critical Besov spaces for the compressible Navier-Stokes equations with density dependent viscosities under the assumption that the initial density is bounded away from zero.
\end{abstract}

\section{Introduction}

In this paper, we consider the compressible Navier-Stokes equations with density dependent viscosities in $\mathbb{R}^{+} \times \mathbb{R}^{N}(N \geq 2)$ :

$$
\left\{\begin{array}{l}
\partial_{t} \rho+\operatorname{div}(\rho u)=0 \\
\partial_{t}(\rho u)+\operatorname{div}(\rho u \otimes u)-\operatorname{div}(2 \mu(\rho) D(u))-\nabla(\lambda(\rho) \operatorname{div} u)+\nabla P(\rho)=0 \\
\left.(\rho, u)\right|_{t=0}=\left(\rho_{0}, u_{0}\right)
\end{array}\right.
$$

Here $\rho(t, x)$ and $u(t, x)$ are the density and velocity of the fluid. The pressure $P$ is a smooth function of $\rho, D(u)=\frac{1}{2}\left(\nabla u+\nabla u^{t}\right)$ is the strain tensor, the Lamé coefficients $\mu$ and $\lambda$ depend smoothly on $\rho$ and satisfy

$$
\mu>0 \text { and } \lambda+2 \mu>0,
$$

which ensures that the operator $-\operatorname{div}(2 \mu(\rho) D \cdot)-\nabla(\lambda(\rho) \operatorname{div} \cdot)$ is elliptic. An important example is included in the system (1.1): the viscous shallow water equations $\left(N=2, \mu(\rho)=\rho, \lambda(\rho)=0\right.$ and $\left.P(\rho)=\rho^{2}\right)$.

2000 Mathematics Subject Classification: 35Q30, 35D10.

Keywords: Compressible Navier-Stokes equations, Besov spaces, Bony's paraproduct, Fourier localization. 
The local existence and uniqueness of smooth solutions for the system (1.1) were proved by Nash [23] for smooth initial data without vacuum. Later on, Matsumura and Nishida [20] proved the global well-posedness for smooth data close to equilibrium, see also [18] for one dimension. Concerning the global existence of weak solutions for the large initial data, we refer to $[2,3,19,21]$. We may refer to $[4,10,25]$ and references therein for the viscous shallow water equations.

This paper is devoted to the study of the well-posedness of the system (1.1) in the critical spaces. Recently, Danchin has obtained several important well-posedness results in the critical spaces for the compressible Navier-Stokes equations $[11,12,14]$. To explain the precise meaning of critical spaces, let us consider the incompressible Navier-Stokes equations

$$
(\mathrm{NS}) \quad\left\{\begin{array}{l}
\partial_{t} u-\Delta u+u \cdot \nabla u+\nabla p=0 \\
\operatorname{div} u=0
\end{array}\right.
$$

It is easy to find that if $(u, p)$ is a solution of $(\mathrm{NS})$, then

$$
u_{\lambda}(t, x) \stackrel{\text { def }}{=} \lambda u\left(\lambda^{2} t, \lambda x\right), \quad p_{\lambda}(t, x) \stackrel{\text { def }}{=} \lambda^{2} p\left(\lambda^{2} t, \lambda x\right)
$$

is also a solution of (NS). For the (NS) equations, a functional space $X$ is critical if the corresponding norm is invariant under the scaling of (1.3). Obviously, $\dot{H}^{\frac{N}{2}-1}$ is a critical space. Fujita and Kato [16] proved the wellposedness of (NS) in $\dot{H}^{\frac{N}{2}-1}$, see also $[5,6,22]$ and references therein for the well-posedness in the other critical spaces. For the compressible NavierStokes equations, let us introduce the following transformation

$$
\rho_{\lambda}(t, x) \stackrel{\text { def }}{=} \rho\left(\lambda^{2} t, \lambda x\right), \quad u_{\lambda}(t, x) \stackrel{\text { def }}{=} \lambda u\left(\lambda^{2} t, \lambda x\right) .
$$

Then if $(\rho, u)$ solves $(1.1)$, so does $\left(\rho_{\lambda}, u_{\lambda}\right)$ provided the viscosity coefficients are constants and the pressure law has been changed into $\lambda^{2} P$. This motivates the following definition:

Definition 1.1 We will say that a functional space is critical with respect to the scaling of the equations if the associated norm is invariant under the transformation:

$$
(\rho, u) \longrightarrow\left(\rho_{\lambda}, u_{\lambda}\right)
$$

(up to a constant independent of $\lambda$ ).

A natural candidate is the homogenous Sobolev space $\dot{H}^{N / 2} \times\left(\dot{H}^{N / 2-1}\right)^{N}$, but since $\dot{H}^{N / 2}$ is not included in $L^{\infty}$, we can not obtain a $L^{\infty}$ control of the density when $\rho_{0} \in \dot{H}^{N / 2}$. 
Instead, we choose the initial data $\left(\rho_{0}, u_{0}\right)$ for some $\overline{\rho_{0}}$ in a critical homogenous Besov spaces:

$$
\left(\rho_{0}-\bar{\rho}_{0}, u_{0}\right) \in \dot{B}_{p, 1}^{\frac{N}{p}} \times\left(\dot{B}_{p, 1}^{\frac{N}{p}-1}\right)^{N},
$$

since $\dot{B}_{p, 1}^{\frac{N}{p}}$ is continuously embedded in $L^{\infty}$.

However, working in the critical spaces, if we deal with the elliptic operators of the momentum equations as a constant coefficient second order operator plus a perturbation induced by the density and viscosity coefficients, the perturbation will be a trouble term. In the case when $\rho-\bar{\rho}_{0}$ is small in $\dot{B}_{p, 1}^{\frac{N}{p}}$ or has more regularity, the perturbation can be treated as a harmless source term and the corresponding local well-posedness can be obtained by following the argument of Danchin [12], see [17].

The purpose of the present paper is to obtain a local well-posedness result in the critical Besov spaces under the natural physical assumption that the initial density is bounded away from zero. Our new observation is that if $\rho-\bar{\rho}_{0}$ is small in the weighted Besov spaces $\dot{B}_{p, 1}^{\frac{N}{p}}(\omega)$ (see Section 3 for the definition), the perturbation can still be treated as a harmless source term. Similar idea has been used by the authors of this paper to prove the local well-posedness in $\dot{B}_{2,1}^{1} \times\left(\dot{B}_{2,1}^{0}\right)^{2}$ for the viscous shallow water equations [10]. Very rencently, Danchin [15] proved a similar result for the system (1.1) with constant coefficients. The key of his proof is a new and interesting estimate for a class of parabolic systems with the coefficients in $C\left([0, T] ; \dot{B}_{2,1}^{N / 2}\right)$. It seems to be possible to adapt his method to the present model. Here we would like to present a general functional framework to deal with the local well-posedness in the critical spaces for the compressible fluids.

Our main result is as follows:

Theorem 1.2 Let $\bar{\rho}_{0}$ and $c_{0}$ be two positive constants. Assume that the initial data satisfies

$$
\left(\rho_{0}-\bar{\rho}_{0}, u_{0}\right) \in \dot{B}_{p, 1}^{\frac{N}{p}} \times\left(\dot{B}_{p, 1}^{\frac{N}{p}-1}\right)^{N} \quad \text { and } \quad \rho_{0} \geq c_{0} .
$$

Then there exists a positive time $T$ such that

(a) Existence: If $p \in(1, N]$, the system (1.1) has a solution $\left(\rho-\bar{\rho}_{0}, u\right)$ $\in E_{T}^{p}$ with

$$
E_{T}^{p} \stackrel{\text { def }}{=} C\left([0, T] ; \dot{B}_{p, 1}^{\frac{N}{p}}\right) \times\left(C\left([0, T] ; \dot{B}_{p, 1}^{\frac{N}{p}-1}\right) \cap L^{1}\left(0, T ; \dot{B}_{p, 1}^{\frac{N}{p}+1}\right)\right)^{N}, \quad \rho \geq \frac{1}{2} c_{0} ;
$$

(b) Uniqueness: If $p \in(1, N]$, then the uniqueness holds in $E_{T}^{p}$. 
Remark 1.3 If the Lamé coefficients $\mu$ and $\lambda$ are constants satisfying (1.2), then the range of $p$ in the existence result of the system (1.1) can be extended to $p \in(1,2 N)$, since we can take $p \in(1,2 N)$ in Proposition 5.1 for the case when $\bar{\lambda}$ and $\bar{\mu}$ are constants.

The structure of this paper is as follows:

In Section 2, we recall some basic facts about the Littlewood-Paley decomposition and the functional spaces. In Section 3, we firstly introduce the weighted Besov spaces, then present some nonlinear estimates. Section 4 is devoted to the estimates in the weighted Besov spaces for the linear transport equation. Section 5 is devoted to the estimates in the weighted Besov spaces for the linearized momentum equation. In Section 6, we prove the existence of the solution. In Section 7, we prove the uniqueness of the solution.

\section{Littlewood-Paley theory and the functional spaces}

Let us introduce the Littlewood-Paley decomposition. Choose a radial function $\varphi \in \mathcal{S}\left(\mathbb{R}^{N}\right)$ supported in $\mathcal{C}=\left\{\xi \in \mathbb{R}^{N}, \frac{3}{4} \leq|\xi| \leq \frac{8}{3}\right\}$ such that

$$
\sum_{j \in \mathbb{Z}} \varphi\left(2^{-j} \xi\right)=1 \quad \text { for all } \xi \neq 0 .
$$

The frequency localization operator $\Delta_{j}$ and $S_{j}$ are defined by

$$
\Delta_{j} f=\varphi\left(2^{-j} D\right) f, \quad S_{j} f=\sum_{k \leq j-1} \Delta_{k} f \quad \text { for } \quad j \in \mathbb{Z} .
$$

With our choice of $\varphi$, one can easily verify that

$$
\begin{aligned}
& \Delta_{j} \Delta_{k} f=0 \quad \text { if }|j-k| \geq 2 \text { and } \\
& \Delta_{j}\left(S_{k-1} f \Delta_{k} f\right)=0 \text { if }|j-k| \geq 5 .
\end{aligned}
$$

We denote the space $\mathcal{Z}^{\prime}\left(\mathbb{R}^{N}\right)$ by the dual space of $\mathcal{Z}\left(\mathbb{R}^{N}\right)=\left\{f \in \mathcal{S}\left(\mathbb{R}^{N}\right)\right.$; $D^{\alpha} \hat{f}(0)=0 ; \forall \alpha \in \mathbb{N}^{N}$ multi-index $\}$, it also can be identified by the quotient space of $\mathcal{S}^{\prime}\left(\mathbb{R}^{N}\right) / \mathcal{P}$ with the polynomials space $\mathcal{P}$. The formal equality

$$
f=\sum_{k \in \mathbb{Z}} \Delta_{k} f
$$

holds true for $f \in \mathcal{Z}^{\prime}\left(\mathbb{R}^{N}\right)$ and is called the homogeneous Littlewood-Paley decomposition.

The operators $\Delta_{j}$ help us recall the definition of the Besov space (see also [24]). 
Definition 2.1 Let $s \in \mathbb{R}, 1 \leq p, r \leq+\infty$. The homogeneous Besov space $\dot{B}_{p, r}^{s}$ is defined by

$$
\dot{B}_{p, r}^{s}=\left\{f \in \mathcal{Z}^{\prime}\left(\mathbb{R}^{N}\right):\|f\|_{\dot{B}_{p, r}^{s}}<+\infty\right\},
$$

where

$$
\|f\|_{\dot{B}_{p, r}^{s}} \stackrel{\text { def }}{=}\left\|2^{k s}\right\| \Delta_{k} f(t)\left\|_{p}\right\|_{\ell^{r}}
$$

We next introduce the Besov-Chemin-Lerner space $\widetilde{L}_{T}^{q}\left(\dot{B}_{p, r}^{s}\right)$ which is initiated in [9].

Definition 2.2 Let $s \in \mathbb{R}, 1 \leq p, q, r \leq+\infty, 0<T \leq+\infty$. The space $\widetilde{L}_{T}^{q}\left(\dot{B}_{p, r}^{s}\right)$ is defined as the set of all the distributions $f$ satisfying

$$
\|f\|_{\widetilde{L}_{T}^{q}\left(\dot{B}_{p, r}^{s}\right)}<+\infty
$$

where

$$
\|f\|_{\widetilde{L}_{T}^{q}\left(\dot{B}_{p, r}^{s}\right)}^{r} \stackrel{\text { def }}{=}\left\|2^{k s}\right\| \Delta_{k} f(t)\left\|_{L^{q}\left(0, T ; L^{p}\right)}\right\|_{\ell^{r}} .
$$

Obviously, $\widetilde{L}_{T}^{1}\left(\dot{B}_{p, 1}^{s}\right)=L_{T}^{1}\left(\dot{B}_{p, 1}^{s}\right)$. In the sequel, we will constantly use the Bony's decomposition from [1] that

$$
u v=T_{u} v+T_{v} u+R(u, v),
$$

with

$$
T_{u} v=\sum_{j \in \mathbb{Z}} S_{j-1} u \Delta_{j} v, \quad R(u, v)=\sum_{j \in \mathbb{Z}} \Delta_{j} u \widetilde{\Delta}_{j} v, \quad \widetilde{\Delta}_{j} v=\sum_{\left|j^{\prime}-j\right| \leq 1} \Delta_{j^{\prime}} v .
$$

Let us conclude this section by collecting some useful lemmas.

Lemma 2.3 ([7]) Let $1 \leq p \leq q \leq+\infty$. Assume that $f \in L^{p}\left(\mathbb{R}^{N}\right)$, then for any $\gamma \in(\mathbb{N} \cup\{0\})^{N}$, there exist constants $C_{1}, C_{2}$ independent of $f, j$ such that

$$
\begin{aligned}
\operatorname{supp} \hat{f} \subseteq\left\{|\xi| \leq A_{0} 2^{j}\right\} & \Longrightarrow\left\|\partial^{\gamma} f\right\|_{q} \leq C_{1} 2^{j|\gamma|+j N\left(\frac{1}{p}-\frac{1}{q}\right)}\|f\|_{p}, \\
\operatorname{supp} \hat{f} \subseteq\left\{A_{1} 2^{j} \leq|\xi| \leq A_{2} 2^{j}\right\} & \Longrightarrow\|f\|_{p} \leq C_{2} 2^{-j|\gamma|} \sup _{|\beta|=|\gamma|}\left\|\partial^{\beta} f\right\|_{p} .
\end{aligned}
$$

Lemma 2.4 ([12]) Let $1<p<\infty$, and $a \geq \bar{a}>0$ be a bounded continuous function. Assume that $u \in L^{p}\left(\mathbb{R}^{N}\right)$ and supp $\hat{u} \subset\left\{\xi: R_{1} \leq|\xi| \leq R_{2}\right\}$. Then there exists a constant $c$ depending only on $N$ and $R_{2} / R_{1}$ such that

$$
c \bar{a} R_{1}^{2} \frac{(p-1)}{p^{2}} \int_{\mathbb{R}^{N}}|u|^{p} \mathrm{~d} x \leq-\int_{\mathbb{R}^{N}} \operatorname{div}(a \nabla u)|u|^{p-2} u \mathrm{~d} x .
$$


Lemma 2.5 Let $s>0$, and $1 \leq p \leq \infty$. Assume that $f, g \in \dot{B}_{p, 1}^{s_{1}} \cap L^{\infty}$. Then there holds

$$
\|f g\|_{\dot{B}_{p, 1}^{s}} \leq C\left(\|f\|_{\dot{B}_{p, 1}^{s}}\|g\|_{L^{\infty}}+\|f\|_{L^{\infty}}\|g\|_{\dot{B}_{p, 1}^{s}}\right) .
$$

Lemma 2.6 Let $s_{1}, s_{2} \leq \frac{N}{p}, s_{1}+s_{2}>N \max \left(0, \frac{2}{p}-1\right)$, and $1 \leq p, q, q_{1}, q_{2} \leq \infty$ with $\frac{1}{q_{1}}+\frac{1}{q_{2}}=\frac{1}{q}$. Assume that $f \in \widetilde{L}_{T}^{q_{1}}\left(\dot{B}_{p, 1}^{s_{1}}\right)$ and $g \in \widetilde{L}_{T}^{q_{2}}\left(\dot{B}_{p, 1}^{s_{2}}\right)$. Then there holds

$$
\|f g\|_{\widetilde{L}_{T}^{q}\left(\dot{B}_{p, 1}^{\left.s_{1}+s_{2}-\frac{N}{p}\right)}\right.} \leq C\|f\|_{\widetilde{L}_{T}^{q_{1}\left(\dot{B}_{p, 1}^{s_{1}}\right)}}\|g\|_{\widetilde{L}_{T}^{q_{2}}\left(\dot{B}_{p, 1}^{s_{2}}\right.} .
$$

Lemma 2.7 Let $s_{1} \leq \frac{N}{p}, s_{2}<\frac{N}{p}, s_{1}+s_{2} \geq N \max \left(0, \frac{2}{p}-1\right)$, and $1 \leq$ $p, q, q_{1}, q_{2} \leq \infty$ with $\frac{1}{q_{1}}+\frac{1}{q_{2}}=\frac{1}{q}$. Assume that $f \in \widetilde{L}_{T}^{q_{1}}\left(\dot{B}_{p, 1}^{s_{1}}\right)$ and $g \in$ $\widetilde{L}_{T}^{q_{2}}\left(\dot{B}_{p, \infty}^{s_{2}}\right)$. Then there holds

$$
\|f g\|_{\widetilde{L}_{T}^{q}\left(\dot{B}_{p, \infty}^{\left.s_{1}+s_{2}-\frac{N}{p}\right)}\right.} \leq C\|f\|_{\widetilde{L}_{T}^{q_{1}\left(\dot{B}_{p, 1}^{s_{1}}\right)}}\|g\|_{\widetilde{L}_{T}^{q_{2}}\left(\dot{B}_{p, \infty}^{s_{2}}\right)}
$$

Lemma 2.8 Let $s \in\left(-N \min \left(\frac{1}{p}, \frac{1}{p^{\prime}}\right), \frac{N}{p}+1\right]$ and $1 \leq p, q, q_{1}, q_{2} \leq \infty$ with $\frac{1}{q_{1}}+\frac{1}{q_{2}}=\frac{1}{q}$. Assume that $f \in \widetilde{L}_{T}^{q_{1}}\left(\dot{B}_{p, 1}^{\frac{N}{p}+1}\right)$ and $g \in \widetilde{L}_{T}^{q_{2}}\left(\dot{B}_{p, 1}^{s}\right)$. Then there
holds

$$
\sum_{j} 2^{j(s-1)}\left\|\operatorname{div}\left[\Delta_{j}, f\right] \nabla g\right\|_{L_{T}^{q}\left(L^{p}\right)} \leq C\|f\|_{\widetilde{L}_{T}^{q_{1}\left(\dot{B}_{p, 1}^{N}\right)}}\|g\|_{\widetilde{L}_{T}^{q_{2}\left(\dot{B}_{p, 1}^{s}\right)}} .
$$

Lemma 2.9 Let $s>0$ and $1 \leq p, q \leq \infty$. Assume that $F \in W_{l o c}^{[s]+3, \infty}(\mathbb{R})$ with $F(0)=0$. Then for any $f \in L_{T}^{\infty}\left(L^{\infty}\right) \cap \widetilde{L}_{T}^{q}\left(\dot{B}_{p, 1}^{s}\right)$, we have

$$
\|F(f)\|_{\tilde{L}_{T}^{q}\left(\dot{B}_{p, 1}^{s}\right)} \leq C\left(1+\|f\|_{L_{T}^{\infty}\left(L^{\infty}\right)}\right)^{[s]+2}\|f\|_{\widetilde{L}_{T}^{q}\left(\dot{B}_{p, 1}^{s}\right)} .
$$

Lemma 2.6-Lemma 2.9 can be easily proved by using Bony's decomposition and Lemma 2.3 , see also $[8,12]$ or Section 3 for similar results.

Remark 2.10 Lemmas 2.6-2.9 still remain true for the usual homogenous Besov spaces. For example, the estimate in Lemma 2.6 becomes

$$
\|f g\|_{\dot{B}_{p, 1}^{s_{1}+s_{2}-\frac{N}{p}}} \leq C\|f\|_{\dot{B}_{p, 1}^{s_{1}}}\|g\|_{\dot{B}_{p, 1}^{s_{2}}}
$$

with $p, s_{1}, s_{2}$ satisfying the conditions as in Lemma 2.6. 


\section{Nonlinear estimates in the weighted Besov spaces}

Let us firstly introduce the weight function. Let $\left\{e_{k}(t)\right\}_{k \in \mathbb{Z}}$ be a sequence defined in $[0,+\infty)$ satisfying the following conditions:

$$
e_{k}(t) \in[0,1], e_{k}(t) \leq e_{k^{\prime}}(t) \text { if } k \leq k^{\prime} \quad \text { and } \quad e_{k}(t) \sim e_{k^{\prime}}(t) \text { if } k \sim k^{\prime}
$$

where $k \sim k^{\prime}$ means that there exists a constant $N_{0}$ such that $\left|k-k^{\prime}\right| \leq N_{0}$. Then the weight function $\left\{\omega_{k}(t)\right\}_{k \in \mathbb{Z}}$ is defined by

$$
\omega_{k}(t)=\sum_{\ell \geq k} 2^{k-\ell} e_{\ell}(t), \quad k \in \mathbb{Z}
$$

It is easy to verify that for any $k \in \mathbb{Z}$,

$$
\left\{\begin{array}{l}
\omega_{k}(t) \leq 2, \quad e_{k}(t) \leq \omega_{k}(t) \\
\omega_{k}(t) \leq 2^{k-k^{\prime}} \omega_{k^{\prime}}(t) \quad \text { if } k \geq k^{\prime}, \quad \omega_{k}(t) \leq 3 \omega_{k^{\prime}}(t) \quad \text { if } k \leq k^{\prime} \\
\omega_{k}(t) \sim \omega_{k^{\prime}}(t) \quad \text { if } k \sim k^{\prime}
\end{array}\right.
$$

Definition 3.1 Let $s \in \mathbb{R}, 1 \leq p, r \leq+\infty, 0<T<+\infty$. The weighted Besov space $\dot{B}_{p, r}^{s}(\omega)$ is defined by

$$
\dot{B}_{p, r}^{s}(\omega)=\left\{f \in \mathcal{Z}^{\prime}\left(\mathbb{R}^{N}\right):\|f\|_{\dot{B}_{p, r}^{s}(\omega)}<+\infty\right\},
$$

where

$$
\|f\|_{\dot{B}_{p, r}^{s}(\omega)} \stackrel{\text { def }}{=}\left\|2^{k s} \omega_{k}(T)\right\| \Delta_{k} f\left\|_{p}\right\|_{\ell^{r}} .
$$

Definition 3.2 Let $s \in \mathbb{R}, 1 \leq p, q \leq+\infty, 0<T<+\infty$. The weighted function space $\widetilde{L}_{T}^{q}\left(\dot{B}_{p, 1}^{s}(\omega)\right)$ is defined by

$$
\widetilde{L}_{T}^{q}\left(\dot{B}_{p, 1}^{s}(\omega)\right)=\left\{f \in L_{T}^{q}\left(\dot{B}_{p, 1}^{s}(\omega)\right):\|f\|_{\widetilde{L}_{T}^{q}\left(\dot{B}_{p, 1}^{s}(\omega)\right)}<+\infty\right\},
$$

where

$$
\|f\|_{\widetilde{L}_{T}^{q}\left(\dot{B}_{p, 1}^{s}(\omega)\right)} \stackrel{\text { def }}{=} \sum_{k \in \mathbb{Z}} 2^{k s} \omega_{k}(T)\left(\int_{0}^{T}\left\|\Delta_{k} f(t)\right\|_{p}^{q} d t\right)^{\frac{1}{q}} .
$$

Remark 3.3 If $e_{k}(t)$ is continuous on $[0,+\infty)$ and $e_{k}(0)=0$ for $k \in \mathbb{Z}$, $f \in \widetilde{L}_{T}^{\infty}\left(\dot{B}_{p, 1}^{s}\right)$, then for any $\varepsilon>0$, there exists a $\widetilde{T} \in(0, T]$ such that

$$
\|f\|_{\widetilde{L}_{\widetilde{T}}^{\infty}\left(\dot{B}_{p, 1}^{s}(\omega)\right)} \leq \varepsilon
$$


Indeed, due to $f \in \widetilde{L}_{T}^{\infty}\left(\dot{B}_{p, 1}^{s}\right)$ and $\omega_{k}(T) \leq 2$, there exists $N_{1} \in \mathbb{N}$ such that

$$
\begin{aligned}
& \sum_{|k| \geq N_{1}+1} 2^{k s} \omega_{k}(T)\left\|\Delta_{k} f\right\|_{L_{T}^{\infty}\left(L^{p}\right)} \leq \varepsilon / 3, \\
& \sum_{|k| \leq N_{1}} 2^{k s} \sum_{\ell \geq k+N_{1}+1} 2^{k-\ell} e_{\ell}(T)\left\|\Delta_{k} f\right\|_{L_{T}^{\infty}\left(L^{p}\right)} \leq \varepsilon / 3 .
\end{aligned}
$$

Thus, we have

$$
\begin{aligned}
\|f\|_{\widetilde{L}_{\widetilde{T}}^{\infty}\left(\dot{B}_{p, 1}^{s}(\omega)\right)} & \leq 2 \varepsilon / 3+\sum_{|k| \leq N_{1}} 2^{k s} \sum_{k \leq \ell \leq k+N_{1}} 2^{k-\ell} e_{\ell}(\widetilde{T})\left\|\Delta_{k} f\right\|_{L_{\widetilde{T}}^{\infty}\left(L^{p}\right)} \\
& \leq 2 \varepsilon / 3+2 e_{2 N_{1}}(\widetilde{T}) \sum_{|k| \leq N_{1}} 2^{k s}\left\|\Delta_{k} f\right\|_{L_{\widetilde{T}}^{\infty}\left(L^{p}\right)} \\
& \leq \varepsilon
\end{aligned}
$$

if $\widetilde{T} \in(0, T]$ is chosen such that

$$
2 e_{2 N_{1}}(\widetilde{T}) \sum_{|k| \leq N_{1}} 2^{k s}\left\|\Delta_{k} f\right\|_{L_{\widetilde{T}}^{\infty}\left(L^{p}\right)} \leq \varepsilon / 3
$$

Next, we present some estimates in the weighted Besov spaces.

Lemma 3.4 Let $1 \leq p \leq \infty$. Assume that $f \in \dot{B}_{p, 1}^{s_{1}}(\omega), g \in \dot{B}_{p, 1}^{s_{2}}$. Then there hold

(a) if $s_{2} \leq \frac{N}{p}$, we have

$$
\left\|T_{g} f\right\|_{\dot{B}_{p, 1}^{s_{1}+s_{2}-\frac{N}{p}(\omega)}} \leq C\|f\|_{\dot{B}_{p, 1}^{s_{1}}(\omega)}\|g\|_{\dot{B}_{p, 1}^{s_{2}}}
$$

(b) if $s_{1} \leq \frac{N}{p}-1$, we have

$$
\left\|T_{f} g\right\|_{\dot{B}_{p, 1}^{s_{1}+s_{2}-\frac{N}{p}}(\omega)} \leq C\|f\|_{\dot{B}_{p, 1}^{s_{1}}(\omega)}\|g\|_{\dot{B}_{p, 1}^{s_{2}}}
$$

(c) if $s_{1}+s_{2}>N \max \left(0, \frac{2}{p}-1\right)$, we have

$$
\|R(f, g)\|_{\dot{B}_{p, 1}^{s_{1}+s_{2}-\frac{N}{p}}(\omega)} \leq C\|f\|_{\dot{B}_{p, 1}^{s_{1}}(\omega)}\|g\|_{\dot{B}_{p, 1}^{s_{2}}} .
$$

Proof. Due to (2.1), we have

$$
\Delta_{j}\left(T_{g} f\right)=\sum_{\left|j^{\prime}-j\right| \leq 4} \Delta_{j}\left(S_{j^{\prime}-1} g \Delta_{j^{\prime}} f\right)
$$


then we get by Lemma 2.3 and (3.2) that

$$
\begin{aligned}
\left\|T_{g} f\right\|_{\dot{B}_{p, 1}^{s_{1}+s_{2}-\frac{N}{p}}(\omega)} & =\sum_{j} 2^{j\left(s_{1}+s_{2}-\frac{N}{p}\right)} \omega_{j}(T)\left\|\Delta_{j}\left(T_{g} f\right)\right\|_{p} \\
& \leq C \sum_{j} 2^{j\left(s_{1}+s_{2}-\frac{N}{p}\right)} \omega_{j}(T)\left\|S_{j-1} g\right\|_{\infty}\left\|\Delta_{j} f\right\|_{p} \\
& \leq C\|f\|_{\dot{B}_{p, 1}^{s_{1}}(\omega)}\|g\|_{\dot{B}_{p, 1}^{s_{2}}},
\end{aligned}
$$

where we used in the last inequality

$$
\left\|S_{j-1} g\right\|_{\infty} \leq C \sum_{\ell \leq j-2} 2^{\ell \frac{N}{p}}\left\|\Delta_{\ell} g\right\|_{p} \leq C 2^{j\left(-s_{2}+\frac{N}{p}\right)}\|g\|_{\dot{B}_{p, 1}^{s_{2}}} .
$$

This proves (a). We next prove (b). Similarly, we have

$$
\begin{aligned}
\left\|T_{f} g\right\|_{\dot{B}_{p, 1}^{s_{1}+s_{2}-\frac{N}{p}}(\omega)} & =\sum_{j} 2^{j\left(s_{1}+s_{2}-\frac{N}{p}\right)} \omega_{j}(T)\left\|\Delta_{j}\left(T_{f} g\right)\right\|_{p} \\
& \leq C \sum_{j} 2^{j\left(s_{1}+s_{2}-\frac{N}{p}\right)} \omega_{j}(T)\left\|S_{j-1} f\right\|_{\infty}\left\|\Delta_{j} g\right\|_{p},
\end{aligned}
$$

and by Lemma 2.3 and (3.2), we have

$$
\begin{aligned}
\omega_{j}(T)\left\|S_{j-1} f\right\|_{\infty} & \leq C 2^{j} \sum_{\ell \leq j-2} 2^{\ell\left(\frac{N}{p}-1\right)} \omega_{\ell}(T)\left\|\Delta_{\ell} f\right\|_{p} \\
& \leq C 2^{j\left(\frac{N}{p}-s_{1}\right)}\|f\|_{\dot{B}_{p, 1}^{s_{1}}(\omega)}
\end{aligned}
$$

which lead to (b). Now we prove (c). Notice that

$$
\Delta_{j}(R(f, g))=\sum_{j^{\prime} \geq j-3} \Delta_{j}\left(\Delta_{j^{\prime}} f \widetilde{\Delta}_{j^{\prime}} g\right)
$$

then we get by Lemma 2.3 that if $p \geq 2$

$$
\begin{aligned}
\|R(f, g)\|_{\dot{B}_{p, 1}^{s_{1}+s_{2}-\frac{N}{p}}(\omega)} & \leq C \sum_{j} \sum_{j^{\prime} \geq j-3} 2^{j\left(s_{1}+s_{2}\right)} \omega_{j}(T)\left\|\Delta_{j^{\prime}} f\right\|_{p}\left\|\widetilde{\Delta}_{j^{\prime}} g\right\|_{p} \\
& \leq C \sum_{j} \sum_{j^{\prime} \geq j-3} \sum_{\ell \geq j} 2^{j-\ell} e_{\ell}(T) 2^{j\left(s_{1}+s_{2}\right)}\left\|\Delta_{j^{\prime}} f\right\|_{p}\left\|\widetilde{\Delta}_{j^{\prime}} g\right\|_{p} \\
& =\sum_{j} \sum_{j^{\prime} \geq j-3} \sum_{\ell \geq j, j^{\prime}} \square+\sum_{j} \sum_{j^{\prime} \geq j-3} \sum_{j^{\prime} \geq \ell \geq j} \square \\
& \triangleq I+I I .
\end{aligned}
$$


For $I I$, using the fact that $e_{\ell}(T) \leq e_{j^{\prime}}(T) \leq \omega_{j^{\prime}}(T)$ if $\ell \leq j^{\prime}$, we get

$$
\begin{aligned}
I I & \leq C \sum_{j} \sum_{j^{\prime} \geq j-3} \omega_{j^{\prime}}(T) 2^{j\left(s_{1}+s_{2}\right)}\left\|\Delta_{j^{\prime}} f\right\|_{p}\left\|\widetilde{\Delta}_{j^{\prime}} g\right\|_{p} \\
& \leq C \sum_{j} \sum_{j^{\prime} \geq j-3} \omega_{j^{\prime}}(T) 2^{\left(j-j^{\prime}\right)\left(s_{1}+s_{2}\right)} 2^{j^{\prime} s_{1}}\left\|\Delta_{j^{\prime}} f\right\|_{p}\|g\|_{\dot{B}_{p, 1}^{s_{2}}} \\
& \leq C\|f\|_{\dot{B}_{p, 1}^{s_{1}}(\omega)}\|g\|_{\dot{B}_{p, 1}^{s_{2}}},
\end{aligned}
$$

and for $I$, using the fact that

$$
\sum_{\ell \geq j, j^{\prime}} 2^{j-\ell} e_{\ell}(T) \leq 2^{j-j^{\prime}} \sum_{\ell \geq j^{\prime}} 2^{j^{\prime}-\ell} e_{\ell}(T)=2^{j-j^{\prime}} w_{j^{\prime}}(T)
$$

we obtain

$$
\begin{aligned}
I & \leq C \sum_{j} \sum_{j^{\prime} \geq j-3} \omega_{j^{\prime}}(T) 2^{j\left(s_{1}+s_{2}\right)} 2^{j-j^{\prime}}\left\|\Delta_{j^{\prime}} f\right\|_{p}\left\|\widetilde{\Delta}_{j^{\prime}} g\right\|_{p} \\
& \leq C \sum_{j} \sum_{j^{\prime} \geq j-3} \omega_{j^{\prime}}(T) 2^{\left(j-j^{\prime}\right)\left(s_{1}+s_{2}+1\right)} 2^{j^{\prime} s_{1}}\left\|\Delta_{j^{\prime}} f\right\|_{p}\|g\|_{\dot{B}_{p, 1}^{s_{2}}} \\
& \leq C\|f\|_{\dot{B}_{p, 1}^{s_{1}(\omega)}}\|g\|_{\dot{B}_{p, 1}^{s_{2}} .}
\end{aligned}
$$

If $p<2$, we get by Lemma 2.3 that

$$
\begin{aligned}
& \|R(f, g)\|_{\dot{B}_{p, 1}^{s_{1}+s_{2}-\frac{N}{p}}(\omega)} \leq \\
& \quad \leq C \sum_{j} \sum_{j^{\prime} \geq j-3} 2^{j\left(s_{1}+s_{2}-N\left(\frac{2}{p}-1\right)\right)} \omega_{j}(T)\left\|\Delta_{j^{\prime}} f\right\|_{p}\left\|\widetilde{\Delta}_{j^{\prime}} g\right\|_{p^{\prime}} \\
& \quad \leq C \sum_{j} \sum_{j^{\prime} \geq j-3} \sum_{\ell \geq j} 2^{j-\ell} e_{\ell}(T) 2^{j\left(s_{1}+s_{2}-N\left(\frac{2}{p}-1\right)\right)}\left\|\Delta_{j^{\prime}} f\right\|_{p}\left\|\widetilde{\Delta}_{j^{\prime}} g\right\|_{p} 2^{N\left(\frac{2}{p}-1\right) j^{\prime}} .
\end{aligned}
$$

Then treating it as in the case of $p \geq 2$, we obtain the same inequality for $s_{1}+s_{2}>N\left(\frac{2}{p}-1\right)$. This proves $(\mathrm{c})$.

We have a similar result in the weighted Besov spaces with the time.

Lemma 3.5 Let $1 \leq p, q, q_{1}, q_{2} \leq \infty$ with $\frac{1}{q_{1}}+\frac{1}{q_{2}}=\frac{1}{q}$. Assume that $f \in$ $\widetilde{L}_{T}^{q_{1}}\left(\dot{B}_{p, 1}^{s_{1}}(\omega)\right), g \in \widetilde{L}_{T}^{q_{2}}\left(\dot{B}_{p, 1}^{s_{2}}\right)$. Then there hold

(a) if $s_{2} \leq \frac{N}{p}$, we have

$$
\left\|T_{g} f\right\|_{\widetilde{L}_{T}^{q}\left(\dot{B}_{p, 1}^{s_{1}+s_{2}-\frac{N}{p}}(\omega)\right)} \leq C\|f\|_{\widetilde{L}_{T}^{q_{1}\left(\dot{B}_{p, 1}^{s_{1}}(\omega)\right)}}\|g\|_{\widetilde{L}_{T}^{q_{2}}\left(\dot{B}_{p, 1}^{s_{2}}\right)} ;
$$


(b) if $s_{1} \leq \frac{N}{p}-1$, we have

$$
\left\|T_{f} g\right\|_{\widetilde{L}_{T}^{q}\left(\dot{B}_{p, 1}^{s_{1}+s_{2}-\frac{N}{p}}(\omega)\right)} \leq C\|f\|_{\widetilde{L}_{T}^{q_{1}\left(\dot{B}_{p, 1}^{s_{1}}(\omega)\right)}}\|g\|_{\widetilde{L}_{T}^{q_{2}\left(\dot{B}_{p, 1}^{s_{2}}\right)}} ;
$$

(c) if $s_{1}+s_{2}>N \max \left(0, \frac{2}{p}-1\right)$, we have

$$
\|R(f, g)\|_{\widetilde{L}_{T}^{q}\left(\dot{B}_{p, 1}^{s_{1}+s_{2}-\frac{N}{p}}(\omega)\right)} \leq C\|f\|_{\widetilde{L}_{T}^{q_{1}}\left(\dot{B}_{p, 1}^{s_{1}}(\omega)\right)}\|g\|_{\widetilde{L}_{T}^{q_{2}}\left(\dot{B}_{p, 1}^{s_{2}}\right)} .
$$

The following proposition is a direct consequence of Lemma 3.5.

Proposition 3.6 Let $s_{1} \leq \frac{N}{p}-1, s_{2} \leq \frac{N}{p}, s_{1}+s_{2}>N \max \left(0, \frac{2}{p}-1\right)$, and $1 \leq p, q, q_{1}, q_{2} \leq \infty$ with $\frac{1}{q_{1}}+\frac{1}{q_{2}}=\frac{1}{q}$. Assume that $f \in \widetilde{L}_{T}^{q_{1}}\left(\dot{B}_{p, 1}^{s_{1}}(\omega)\right)$ and $g \in \widetilde{L}_{T}^{q_{2}}\left(\dot{B}_{p, 1}^{s_{2}}\right)$. Then there holds

$$
\|f g\|_{\widetilde{L}_{T}^{q}\left(\dot{B}_{p, 1}^{s_{1}+s_{2}-\frac{N}{p}}(\omega)\right)} \leq C\|f\|_{\widetilde{L}_{T}^{q_{1}}\left(\dot{B}_{p, 1}^{s_{1}}(\omega)\right)}\|g\|_{\widetilde{L}_{T}^{q_{2}}\left(\dot{B}_{p, 1}^{s_{2}}\right)} .
$$

From the proof of Lemma 3.4, we can also obtain

Proposition 3.7 Let $s_{1} \leq \frac{N}{p}-1, s_{2}<\frac{N}{p}, s_{1}+s_{2} \geq N \max \left(0, \frac{2}{p}-1\right)$, and $1 \leq p, q, q_{1}, q_{2} \leq \infty$ with $\frac{1}{q_{1}}+\frac{1}{q_{2}}=\frac{1}{q}$. Assume that $f \in \widetilde{L}_{T}^{q_{1}}\left(\dot{B}_{p, 1}^{s_{1}}(\omega)\right)$ and $g \in \widetilde{L}_{T}^{q_{2}}\left(\dot{B}_{p, \infty}^{s_{2}}\right)$. Then there holds

$$
\|f g\|_{\widetilde{L}_{T}^{q}\left(\dot{B}_{p, \infty}^{s_{1}+s_{2}-\frac{N}{p}}(\omega)\right)} \leq C\|f\|_{\widetilde{L}_{T}^{q_{1}}\left(\dot{B}_{p, 1}^{s_{1}}(\omega)\right)}\|g\|_{\widetilde{L}_{T}^{q_{2}}\left(\dot{B}_{p, \infty}^{s_{2}}\right)} .
$$

Proposition 3.8 Let $s>0$ and $1 \leq p, q \leq \infty$. Assume that $F \in W_{l o c}^{[s]+3, \infty}(\mathbb{R})$ with $F(0)=0$. Then for any $f \in L_{T}^{\infty}\left(L^{\infty}\right) \cap \widetilde{L}_{T}^{q}\left(\dot{B}_{p, 1}^{s}(\omega)\right)$, we have

$$
\|F(f)\|_{\widetilde{L}_{T}^{q}\left(\dot{B}_{p, 1}^{s}(\omega)\right)} \leq C\left(1+\|f\|_{L_{T}^{\infty}\left(L^{\infty}\right)}\right)^{[s]+2}\|f\|_{\widetilde{L}_{T}^{q}\left(\dot{B}_{p, 1}^{s}(\omega)\right)} .
$$

Proof. We decompose $F(f)$ as

$$
\begin{aligned}
F(f)=\sum_{j^{\prime} \in \mathbb{Z}} F\left(S_{j^{\prime}+1} f\right)-F\left(S_{j^{\prime}} f\right) & =\sum_{j^{\prime} \in \mathbb{Z}} \Delta_{j^{\prime}} f \int_{0}^{1} F^{\prime}\left(S_{j^{\prime}} f+\tau \Delta_{j^{\prime}} f\right) d \tau \\
& \triangleq \sum_{j^{\prime} \in \mathbb{Z}} \Delta_{j^{\prime}} f m_{j^{\prime}}(f),
\end{aligned}
$$

where $m_{j^{\prime}}(f)=\int_{0}^{1} F^{\prime}\left(S_{j^{\prime}} f+\tau \Delta_{j^{\prime}} f\right) d \tau$. Furthermore, we write

$$
\Delta_{j} F(f)=\sum_{j^{\prime}<j} \Delta_{j}\left(\Delta_{j^{\prime}} f m_{j^{\prime}}(f)\right)+\sum_{j^{\prime} \geq j} \Delta_{j}\left(\Delta_{j^{\prime}} f m_{j^{\prime}}(f)\right) \triangleq I+I I .
$$


By Lemma 2.3, we have

$$
\begin{aligned}
\|I\|_{L_{T}^{q}\left(L^{p}\right)} & \leq \sum_{j^{\prime}<j}\left\|\Delta_{j}\left(\Delta_{j^{\prime}} f m_{j^{\prime}}(f)\right)\right\|_{L_{T}^{q}\left(L^{p}\right)} \\
& \leq \sum_{j^{\prime}<j} 2^{-j|\alpha|} \sup _{|\gamma|=|\alpha|}\left\|D^{\gamma} \Delta_{j}\left(\Delta_{j^{\prime}} f m_{j^{\prime}}(f)\right)\right\|_{L_{T}^{q}\left(L^{p}\right)},
\end{aligned}
$$

with $\alpha$ to be determined later. Notice that for $|\gamma| \geq 0$, we have

$$
\left\|D^{\gamma} m_{j^{\prime}}(f)\right\|_{\infty} \leq C 2^{j^{\prime}|\gamma|}\left(1+\|f\|_{\infty}\right)^{|\gamma|}\left\|F^{\prime}\right\|_{W|\gamma|, \infty},
$$

from which and (3.3), it follows that

$$
2^{j s}\|I\|_{L_{T}^{q}\left(L^{p}\right)} \leq C 2^{j(s-|\alpha|)} \sum_{j^{\prime}<j} 2^{j^{\prime}|\alpha|}\left\|\Delta_{j^{\prime}} f\right\|_{L_{T}^{q}\left(L^{p}\right)}\left(1+\|f\|_{L_{T}^{\infty}\left(L^{\infty}\right)}\right)^{|\alpha|}\left\|F^{\prime}\right\|_{W^{|\alpha|, \infty}},
$$

thus, if we take $|\alpha|=[s]+2$, we get by (3.2) that

$$
\begin{aligned}
\sum_{j} & \omega_{j}(T) 2^{j s}\|I\|_{L_{T}^{q}\left(L^{p}\right)} \leq \\
\leq & C \sum_{j^{\prime}} 2^{j^{\prime} s} \omega_{j^{\prime}}(T)\left\|\Delta_{j^{\prime}} f\right\|_{L_{T}^{q}\left(L^{p}\right)} \times \\
& \times \sum_{j>j^{\prime}} 2^{\left(j-j^{\prime}\right)(s-|\alpha|+1)}\left(1+\|f\|_{L_{T}^{\infty}\left(L^{\infty}\right)}\right)^{|\alpha|}\left\|F^{\prime}\right\|_{W^{|\alpha|, \infty}} \\
\leq & C\left(1+\|f\|_{L_{T}^{\infty}\left(L^{\infty}\right)}\right)^{[s]+2}\left\|F^{\prime}\right\|_{W^{[s]+2, \infty}}\|f\|_{\widetilde{L}_{T}^{q}\left(\dot{B}_{p, 1}^{s}(\omega)\right)} .
\end{aligned}
$$

Now, let us turn to the estimate of $I I$. We get by Lemma 2.3 that

$$
\|I I\|_{L_{T}^{q}\left(L^{p}\right)} \leq C \sum_{j^{\prime} \geq j}\left\|\Delta_{j^{\prime}} f\right\|_{L_{T}^{q}\left(L^{p}\right)}
$$

Then we write

$$
\begin{aligned}
\sum_{j} \omega_{j}(T) 2^{j s}\|I I\|_{L_{T}^{q}\left(L^{p}\right)} \leq & C \sum_{j} 2^{j s} \sum_{j^{\prime} \geq j}\left\|\Delta_{j^{\prime}} f\right\|_{L_{T}^{q}\left(L^{p}\right)} \sum_{j^{\prime} \geq \ell \geq j} 2^{j-\ell} e_{\ell}(T) \\
& +C \sum_{j} 2^{j s} \sum_{j^{\prime} \geq j}\left\|\Delta_{j^{\prime}} f\right\|_{L_{T}^{q}\left(L^{p}\right)} \sum_{\ell \geq j, j^{\prime}} 2^{j-\ell} e_{\ell}(T),
\end{aligned}
$$

from which and a similar argument of (c) in Lemma 3.4, we infer that

$$
\sum_{j} \omega_{j}(T) 2^{j s}\|I I\|_{L_{T}^{q}\left(L^{p}\right)} \leq C\|f\|_{\widetilde{L}_{T}^{q}\left(\dot{B}_{p, 1}^{s}(\omega)\right)}
$$

from which and (3.4), we conclude the proof of Proposition 3.8. 


\section{Estimates of the linear transport equation}

In this section, we study the linear transport equation

$$
\left\{\begin{array}{l}
\partial_{t} f+v \cdot \nabla f=g \\
f(0, x)=f_{0}
\end{array}\right.
$$

Proposition 4.1 [14] Let $s \in\left(-N \min \left(\frac{1}{p}, \frac{1}{p^{\prime}}\right), 1+\frac{N}{p}\right), 1 \leq p, r \leq+\infty$, and $s=1+\frac{N}{p}$, if $r=1$. Let $v$ be a vector field such that $\nabla v \in L_{T}^{1}\left(\dot{B}_{p, r}^{\frac{N}{p}} \cap L^{\infty}\right)$. Assume that $f_{0} \in \dot{B}_{p, r}^{s}, g \in L_{T}^{1}\left(\dot{B}_{p, r}^{s}\right)$ and $f$ is the solution of (4.1). Then there holds for $t \in[0, T]$,

$$
\|f\|_{\widetilde{L}_{t}^{\infty}\left(\dot{B}_{p, r}^{s}\right)} \leq e^{C V(t)}\left(\left\|f_{0}\right\|_{\dot{B}_{p, r}^{s}}+\int_{0}^{t} e^{-C V(\tau)}\|g(\tau)\|_{\dot{B}_{p, r}^{s}} d \tau\right)
$$

where $V(t) \stackrel{\text { def }}{=} \int_{0}^{t}\|\nabla v(\tau)\|_{\dot{B}_{p, r}^{\frac{N}{p}} \cap L^{\infty}} d \tau$. If $r<+\infty$, then f belongs to $C\left([0, T] ; \dot{B}_{p, r}^{s}\right)$.

Proposition 4.2 Let $p \in[1,+\infty]$ and $s \in\left(-N \min \left(\frac{1}{p}, \frac{1}{p^{\prime}}\right), \frac{N}{p}\right]$. Let $v$ be a vector field such that $\nabla v \in L_{T}^{1}\left(\dot{B}_{p, 1}^{\frac{N}{p}}\right)$. Assume that $f_{0} \in \dot{B}_{p, 1}^{s}, g \in L_{T}^{1}\left(\dot{B}_{p, 1}^{s}\right)$ and $f$ is the solution of (4.1). Then there holds for $t \in[0, T]$,

$$
\|f\|_{\widetilde{L}_{t}^{\infty}\left(\dot{B}_{p, 1}^{s}(\omega)\right)} \leq e^{C V(t)}\left(\left\|f_{0}\right\|_{\dot{B}_{p, 1}^{s}(\omega)}+\int_{0}^{t} e^{-C V(\tau)}\|g(\tau)\|_{\dot{B}_{p, 1}^{s}(\omega)} d \tau\right),
$$

where $V(t) \stackrel{\text { def }}{=} \int_{0}^{t}\|\nabla v(\tau)\|_{\dot{B}_{p, 1}^{\frac{N}{p}}} d \tau$.

Proof. Applying the operator $\Delta_{j}$ to the transport equation, we obtain

$$
\partial_{t} \Delta_{j} f+v \cdot \nabla \Delta_{j} f=\Delta_{j} g+\left[v, \Delta_{j}\right] \cdot \nabla f .
$$

Assume that $p<+\infty$. Multiplying both sides of (4.2) by $\left|\Delta_{j} f\right|^{p-2} \Delta_{j} f$, we get by integrating by parts over $\mathbb{R}^{N}$ for the resulting equation that

$$
\frac{1}{p} \frac{d}{d t}\left\|\Delta_{j} f\right\|_{p}^{p}-\frac{1}{p} \int_{\mathbb{R}^{N}}\left|\Delta_{j} f\right|^{p} \operatorname{div} v d x \leq\left(\left\|\Delta_{j} g\right\|_{p}+\left\|\left[v, \Delta_{j}\right] \cdot \nabla f\right\|_{p}\right)\left\|\Delta_{j} f\right\|_{p}^{p-1},
$$

then we have

$$
\left\|\Delta_{j} f(t)\right\|_{p} \leq\left\|\Delta_{j} f_{0}\right\|_{p}+\int_{0}^{t}\left(\left\|\Delta_{j} g\right\|_{p}+\left\|\left[v, \Delta_{j}\right] \cdot \nabla f\right\|_{p}+\frac{1}{p}\|\operatorname{div} v\|_{\infty}\left\|\Delta_{j} f\right\|_{p}\right) d \tau
$$


from which, it follows that

$$
\begin{aligned}
\|f\|_{\widetilde{L}_{t}^{\infty}\left(\dot{B}_{p, 1}^{s}(\omega)\right)} \leq & \left\|f_{0}\right\|_{\dot{B}_{p, 1}^{s}(\omega)}+C \int_{0}^{t}\|\operatorname{div} v(\tau)\|_{\dot{B}_{p, 1}^{\frac{N}{p}}}\|f(\tau)\|_{\widetilde{L}_{\tau}^{\infty}\left(\dot{B}_{p, 1}^{s}(\omega)\right)} d \tau \\
& +\int_{0}^{t}\|g(\tau)\|_{\dot{B}_{p, 1}^{s}(\omega)} d \tau+\int_{0}^{t} \sum_{j} \omega_{j}(T) 2^{j s}\left\|\left[v, \Delta_{j}\right] \cdot \nabla f(\tau)\right\|_{p} d \tau,
\end{aligned}
$$

from which and Lemma 4.3, we infer that

$$
\begin{aligned}
\|f\|_{\widetilde{L}_{t}^{\infty}\left(\dot{B}_{p, 1}^{s}(\omega)\right)} \leq\left\|f_{0}\right\|_{\dot{B}_{p, 1}^{s}(\omega)} & +C \int_{0}^{t}\|v(\tau)\|_{\dot{B}_{p, 1}^{\frac{N}{p}+1}}\|f(\tau)\|_{\widetilde{L}_{\tau}^{\infty}\left(\dot{B}_{p, 1}^{s}(\omega)\right)} d \tau \\
& +\int_{0}^{t}\|g(\tau)\|_{\dot{B}_{p, 1}^{s}(\omega)} d \tau
\end{aligned}
$$

Then Gronwall's lemma applied implies the desired inequality.

Lemma 4.3 Let $p \in[1, \infty], s \in\left(-N \min \left(\frac{1}{p}, \frac{1}{p^{\prime}}\right), \frac{N}{p}\right]$. Assume that $v \in \dot{B}_{p, 1}^{\frac{N}{p}+1}$ and $f \in \dot{B}_{p, 1}^{s}(\omega)$. Then there holds

$$
\sum_{j} \omega_{j}(T) 2^{j s}\left\|\left[v, \Delta_{j}\right] \cdot \nabla f\right\|_{p} \leq C\|v\|_{\dot{B}_{p, 1}^{\frac{N}{p}+1}}\|f\|_{\dot{B}_{p, 1}^{s}(\omega)} \cdot
$$

Proof. Using the Bony's decomposition, we write

$$
\begin{aligned}
{\left[v, \Delta_{j}\right] \cdot \nabla f=} & {\left[T_{v^{k}}, \Delta_{j}\right] \partial_{k} f+T_{\partial_{k} \Delta_{j} f} v^{k}+R\left(v^{k}, \partial_{k} \Delta_{j} f\right) } \\
& -\Delta_{j}\left(T_{\partial_{k} f} v^{k}\right)-\Delta_{j} R\left(v^{k}, \partial_{k} f\right) .
\end{aligned}
$$

Using Lemma 3.4 with $s_{1}=s-1$ and $s_{2}=\frac{N}{p}+1$, we get

$$
\begin{gathered}
\sum_{j} \omega_{j}(T) 2^{j s}\left\|\Delta_{j}\left(T_{\partial_{k} f} v^{k}\right)\right\|_{p} \leq C\|v\|_{\dot{B}_{p, 1}^{\frac{N}{p}+1}}\|f\|_{\dot{B}_{p, 1}^{s}(\omega)}, \\
\sum_{j} \omega_{j}(T) 2^{j s}\left\|\Delta_{j} R\left(v^{k}, \partial_{k} f\right)\right\|_{p} \leq C\|v\|_{\dot{B}_{p, 1}^{\frac{N}{p}+1}}\|f\|_{\dot{B}_{p, 1}^{s}(\omega)} .
\end{gathered}
$$

Notice that

$$
T_{\partial_{k} \Delta_{j} f}^{\prime} v^{k} \triangleq T_{\partial_{k} \Delta_{j} f} v^{k}+R\left(v^{k}, \partial_{k} \Delta_{j} f\right)=\sum_{j^{\prime} \geq j-2} S_{j^{\prime}+2} \Delta_{j} \partial_{k} f \Delta_{j^{\prime}} v^{k},
$$

then we get by Lemma 2.3 that

$$
\begin{aligned}
\sum_{j} \omega_{j}(T) 2^{j s}\left\|T_{\partial_{k} \Delta_{j} f}^{\prime} v^{k}\right\|_{p} & \leq C \sum_{j} \omega_{j}(T) 2^{j s}\left\|\Delta_{j} \nabla f\right\|_{\infty} \sum_{j^{\prime} \geq j-2}\left\|\Delta_{j^{\prime}} v^{k}\right\|_{p} \\
& \leq C \sum_{j} \omega_{j}(T) 2^{j\left(s+1+\frac{N}{p}\right)}\left\|\Delta_{j} f\right\|_{p} \sum_{j^{\prime} \geq j}\left\|\Delta_{j^{\prime}} v^{k}\right\|_{p} \\
& \leq C\|v\|_{\dot{B}_{p, 1}^{\frac{N}{p}+1}}\|f\|_{\dot{B}_{p, 1}^{s}(\omega)}
\end{aligned}
$$


Now, we turn to estimate $\left[T_{v^{k}}, \Delta_{j}\right] \partial_{k} f$. Set $h(x)=\left(\mathcal{F}^{-1} \varphi\right)(x)$, we get by using Taylor's formula that

$$
\begin{aligned}
& {\left[T_{v^{k}}, \Delta_{j}\right] \partial_{k} f=\sum_{\left|j^{\prime}-j\right| \leq 4}\left[S_{j^{\prime}-1} v^{k}, \Delta_{j}\right] \partial_{k} \Delta_{j^{\prime}} f} \\
& =\sum_{\left|j^{\prime}-j\right| \leq 4} 2^{N j} \int_{\mathbb{R}^{N}} h\left(2^{j}(x-y)\right)\left(S_{j^{\prime}-1} v^{k}(x)-S_{j^{\prime}-1} v^{k}(y)\right) \partial_{k} \Delta_{j^{\prime}} f(y) d y \\
& =\sum_{\left|j^{\prime}-j\right| \leq 4} 2^{(N+1) j} \int_{\mathbb{R}^{N}} \int_{0}^{1} y \cdot \nabla S_{j^{\prime}-1} v^{k}(x-\tau y) d \tau \partial_{k} h\left(2^{j} y\right) \Delta_{j^{\prime}} f(x-y) d y \\
& \quad+2^{N j} \int_{\mathbb{R}^{N}} h\left(2^{j}(x-y)\right) \partial_{k} S_{j^{\prime}-1} v^{k}(y) \Delta_{j^{\prime}} f(y) d y,
\end{aligned}
$$

from which and the Minkowski inequality, we infer that

$$
\begin{aligned}
\sum_{j} \omega_{j}(T) 2^{j s}\left\|\left[T_{v^{k}}, \Delta_{j}\right] \partial_{k} f\right\|_{p} & \leq C \sum_{j} \omega_{j}(T) 2^{j s} \sum_{\left|j^{\prime}-j\right| \leq 4}\left\|\nabla S_{j^{\prime}-1} v\right\|_{\infty}\left\|\Delta_{j^{\prime}} f\right\|_{p} \\
& \leq C\|v\|_{\dot{B}_{p, 1}^{\frac{N}{p}+1}}\|f\|_{\dot{B}_{p, 1}^{s}(\omega)} .
\end{aligned}
$$

Summing up the above estimates, we conclude the proof of Lemma 4.3.

\section{Estimates of the linearized momentum equation}

In this section, we study the linearized momentum equation

$$
\left\{\begin{array}{l}
\partial_{t} u-\operatorname{div}(\bar{\mu} \nabla u)-\nabla((\bar{\lambda}+\bar{\mu}) \operatorname{div} u)=G \\
\left.u\right|_{t=0}=u_{0}
\end{array}\right.
$$

In what follows, we assume that the viscosity coefficients $\bar{\lambda}(\rho)$ and $\bar{\mu}(\rho)$ depend smoothly on the function $\rho$ and there exists a positive constant $c_{1}$ such that

$$
\bar{\mu} \geq c_{1}, \quad \bar{\lambda}+2 \bar{\mu} \geq c_{1} .
$$

Fix a positive constant $c$ to be chosen later. In this section, the weighted function $\omega_{k}(t)$ is given by

$$
\omega_{k}(t)=\sum_{\ell \geq k} 2^{k-\ell} e_{\ell}(t)
$$

with $e_{\ell}(t)=\left(1-e^{-c 2^{2 \ell} t}\right)^{\frac{1}{2}}$. It is easy to verify that the function $e_{\ell}(t)$ satisfies (3.1). 
Proposition 5.1 Let $q \in[1, \infty]$. Assume that $G \in L_{T}^{1}\left(\dot{B}_{p, 1}^{s-1}\right), u_{0} \in \dot{B}_{p, 1}^{s-1}$, and $\rho-\underline{\rho} \in L_{T}^{\infty}\left(\dot{B}_{p, 1}^{\frac{N}{p}}\right)$. Let $u$ be a solution of (5.1). Then there hold (a) If $p \in(1, N], s \in\left(-N \min \left(\frac{1}{p}, \frac{1}{p^{\prime}}\right)+1, \frac{N}{p}\right]$, we have

$$
\begin{aligned}
& \|u\|_{\widetilde{L}_{T}^{q}\left(\dot{B}_{p, 1}^{s-1+2 / q}\right)} \leq \\
& \quad \leq C\left(\left\|u_{0}\right\|_{\dot{B}_{p, 1}^{s-1}}+\|G(\tau)\|_{\widetilde{L}_{T}^{1}\left(\dot{B}_{p, 1}^{s-1}\right)}+A(T)\|\rho-\underline{\rho}\|_{\widetilde{L}_{T}^{\infty}\left(\dot{B}_{p, 1}^{\frac{N}{p}}\right)}\|u\|_{\widetilde{L}_{T}^{1}\left(\dot{B}_{p, 1}^{s+1}\right)}\right)
\end{aligned}
$$

In addition, if $\rho-\underline{\rho} \in L_{T}^{\infty}\left(\dot{B}_{p, 1}^{\frac{N}{p}+1}\right), p \in(1, \infty), s \in\left(-N \min \left(\frac{1}{p}, \frac{1}{p^{\prime}}\right)+1, \frac{N}{p}+1\right]$, then

$$
\begin{aligned}
& \|u\|_{\widetilde{L}_{T}^{q}\left(\dot{B}_{p, 1}^{s-1+2 / q}\right)} \leq \\
& \quad \leq C\left(\left\|u_{0}\right\|_{\dot{B}_{p, 1}^{s-1}}+\|G(\tau)\|_{\widetilde{L}_{T}^{1}\left(\dot{B}_{p, 1}^{s-1}\right)}+A(T)\|\rho-\underline{\rho}\|_{\widetilde{L}_{T}^{\infty}\left(\dot{B}_{p, 1}^{\frac{N}{p}+1}\right)}\|u\|_{\widetilde{L}_{T}^{1}\left(\dot{B}_{p, 1}^{s}\right)}\right)
\end{aligned}
$$

(b) If $p \in(1, N], s \in\left(-N \min \left(\frac{1}{p}, \frac{1}{p^{\prime}}\right)+1, \frac{N}{p}\right]$, we have

$$
\begin{aligned}
& \|u\|_{\widetilde{L}_{T}^{1}\left(\dot{B}_{p, 1}^{s+1}\right)}+\|u\|_{\widetilde{L}_{T}^{2}\left(\dot{B}_{p, 1}^{s}\right)} \leq \\
& \quad \leq C\left(\left\|u_{0}\right\|_{\left.\dot{B}_{p, 1}^{s-1}(\omega)\right)}+\|G(\tau)\|_{\widetilde{L}_{T}^{1}\left(\dot{B}_{p, 1}^{s-1}(\omega)\right)}+A(T)\|\rho-\underline{\rho}\|_{\widetilde{L}_{T}^{\infty}\left(\dot{B}_{p, 1}^{\frac{N}{p}}(\omega)\right)}\|u\|_{\widetilde{L}_{T}^{1}\left(\dot{B}_{p, 1}^{s+1}\right)}\right) .
\end{aligned}
$$

Here $A(T) \stackrel{\text { def }}{=}\left(1+\|\rho\|_{L_{T}^{\infty}\left(L^{\infty}\right)}\right)^{\left[\frac{N}{p}\right]+2}$.

Proof. Set $d=\operatorname{div} u$ and $w=\operatorname{curl} u$. From (5.1), we find that $(d, w)$ satisfies

$$
\left\{\begin{array}{l}
\partial_{t} d-\operatorname{div}(\bar{\nu} \nabla d)=\operatorname{div} G+F_{1} \\
\partial_{t} w-\operatorname{div}(\bar{\mu} \nabla w)=\operatorname{curl} G+F_{2}, \\
\left.(d, w)\right|_{t=0}=\left(\operatorname{div} u_{0}, \operatorname{curl} u_{0}\right) \triangleq\left(d_{0}, w_{0}\right)
\end{array}\right.
$$

where $\bar{\nu}=\bar{\lambda}+2 \bar{\mu}$ and

$$
\begin{aligned}
F_{1} & =\operatorname{div}(\nabla \bar{\mu} \cdot \nabla u)+\operatorname{div}(\nabla(\bar{\lambda}+\bar{\mu}) d), \\
F_{2}^{i, j} & =\operatorname{div}\left(\partial_{j} \bar{\mu} \nabla u^{i}-\partial_{i} \bar{\mu} \nabla u^{j}\right), \quad i, j=1, \ldots, N .
\end{aligned}
$$

Applying the operator $\Delta_{j}$ to $(5.2)$, we obtain

$$
\left\{\begin{array}{l}
\partial_{t} \Delta_{j} d-\operatorname{div}\left(\bar{\nu} \nabla \Delta_{j} d\right)=\operatorname{div} \Delta_{j} G+\Delta_{j} F_{1}+\operatorname{div}\left[\Delta_{j}, \bar{\nu}\right] \nabla d, \\
\partial_{t} \Delta_{j} w-\operatorname{div}\left(\bar{\mu} \nabla \Delta_{j} w\right)=\operatorname{curl} \Delta_{j} G+\Delta_{j} F_{2}+\operatorname{div}\left[\Delta_{j}, \bar{\mu}\right] \nabla w
\end{array}\right.
$$


Multiplying the first equation by $\left|\Delta_{j} d\right|^{p-2} \Delta_{j} d$, we get by integrating over $\mathbb{R}^{N}$ that

$$
\begin{aligned}
\frac{1}{p} \frac{\mathrm{d}}{\mathrm{d} t}\left\|\Delta_{j} d\right\|_{p}^{p}-\int_{\mathbb{R}^{N}} \operatorname{div}\left(\bar{\nu} \nabla \Delta_{j} d\right)\left|\Delta_{j} d\right|^{p-2} \Delta_{j} d \mathrm{~d} x= \\
\quad=\int_{\mathbb{R}^{N}}\left(\operatorname{div} \Delta_{j} G+\Delta_{j} F_{1}+\operatorname{div}\left[\Delta_{j}, \bar{\nu}\right] \nabla d\right)\left|\Delta_{j} d\right|^{p-2} \Delta_{j} d \mathrm{~d} x
\end{aligned}
$$

Lemma 2.4 ensures there exists a positive constant $c_{p}$ depending on $c_{0}, p, N$ such that

$$
\begin{aligned}
\frac{1}{p} \frac{\mathrm{d}}{\mathrm{d} t}\left\|\Delta_{j} d\right\|_{p}^{p} & +c_{p} 2^{2 j}\left\|\Delta_{j} d\right\|_{p}^{p} \leq \\
& \leq\left\|\Delta_{j} d\right\|_{p}^{p-1}\left(\left\|\operatorname{div} \Delta_{j} G\right\|_{p}+\left\|\Delta_{j} F_{1}\right\|_{p}+\left\|\operatorname{div}\left[\Delta_{j}, \bar{\nu}\right] \nabla d\right\|_{p}\right) .
\end{aligned}
$$

Thus, we have

$$
\frac{\mathrm{d}}{\mathrm{d} t}\left\|\Delta_{j} d\right\|_{p}+c_{p} 2^{2 j}\left\|\Delta_{j} d\right\|_{p} \leq\left\|\operatorname{div} \Delta_{j} G\right\|_{p}+\left\|\Delta_{j} F_{1}\right\|_{p}+\left\|\operatorname{div}\left[\Delta_{j}, \bar{\nu}\right] \nabla d\right\|_{p}
$$

which implies that

$$
\begin{gathered}
\left\|\Delta_{j} d(t)\right\|_{p} \leq e^{-c_{p} 2^{2 j} t}\left\|\Delta_{j} d_{0}\right\|_{p}+\int_{0}^{t} e^{-c_{p} 2^{2 j}(t-\tau)}\left(\left\|\operatorname{div} \Delta_{j} G\right\|_{p}+\left\|\Delta_{j} F_{1}\right\|_{p}\right. \\
\left.+\left\|\operatorname{div}\left[\Delta_{j}, \bar{\nu}\right] \nabla d\right\|_{p}\right) \mathrm{d} \tau .
\end{gathered}
$$

Similarly, we can obtain

$$
\begin{aligned}
\left\|\Delta_{j} w(t)\right\|_{p} \leq e^{-c_{p} 2^{2 j} t}\left\|\Delta_{j} w_{0}\right\|_{p}+ & \int_{0}^{t} e^{-c_{p} 2^{2 j}(t-\tau)}\left(\left\|\operatorname{curl} \Delta_{j} G\right\|_{p}\right. \\
& \left.+\left\|\Delta_{j} F_{2}\right\|_{p}+\left\|\operatorname{div}\left[\Delta_{j}, \bar{\mu}\right] \nabla w\right\|_{p}\right) \mathrm{d} \tau .
\end{aligned}
$$

From the above two inequalities, we infer that for any $q \in[1, \infty]$ and $t \in[0, T]$,

$$
\begin{aligned}
& \left\|\Delta_{j} d(t)\right\|_{L_{t}^{q}\left(L^{p}\right)}+\left\|\Delta_{j} w(t)\right\|_{L_{t}^{q}\left(L^{p}\right)} \leq \\
& \quad \leq C 2^{-2 j / q} c_{j}(T)^{\frac{1}{q}}\left(\left\|\Delta_{j} d_{0}\right\|_{p}+\left\|\Delta_{j} w_{0}\right\|_{p}\right) \\
& \quad+C 2^{-2 j / q} c_{j}(T)^{\frac{1}{q}}\left(\left\|\operatorname{div} \Delta_{j} G\right\|_{L_{t}^{1}\left(L^{p}\right)}+\left\|\Delta_{j} F_{1}\right\|_{L_{t}^{1}\left(L^{p}\right)}+\left\|\operatorname{div}\left[\Delta_{j}, \bar{\nu}\right] \nabla d\right\|_{L_{t}^{1}\left(L^{p}\right)}\right) \\
& \quad+C 2^{-2 j / q} c_{j}(T)^{\frac{1}{q}}\left(\left\|\operatorname{curl} \Delta_{j} G\right\|_{L_{t}^{1}\left(L^{p}\right)}+\left\|\Delta_{j} F_{2}\right\|_{L_{t}^{1}\left(L^{p}\right)}+\left\|\operatorname{div}\left[\Delta_{j}, \bar{\mu}\right] \nabla w\right\|_{L_{t}^{1}\left(L^{p}\right)}\right),
\end{aligned}
$$

with $c_{j}(T)=1-e^{c_{p} 2^{2 j} T}$. Notice that

$$
2^{j}\left\|\Delta_{j} u\right\|_{p} \sim\left\|\Delta_{j} d\right\|_{p}+\left\|\Delta_{j} w\right\|_{p}, \quad e_{j}(T) \leq \omega_{j}(T),
$$


which together with (5.3) implies that

$$
\begin{aligned}
& \|u\|_{\widetilde{L}_{T}^{q}\left(\dot{B}_{p, 1}^{s-1+2 / q}\right)} \leq C\left(\left\|u_{0}\right\|_{\dot{B}_{p, 1}^{s-1}}+\|G\|_{\widetilde{L}_{T}^{1}\left(\dot{B}_{p, 1}^{s-1}\right)}+\left\|\left(F_{1}, F_{2}\right)\right\|_{\widetilde{L}_{T}^{1}\left(\dot{B}_{p, 1}^{s-2}\right)}\right) \\
& \quad+C \sum_{j} 2^{j(s-2)}\left(\left\|\operatorname{div}\left[\Delta_{j}, \bar{\nu}\right] \nabla d\right\|_{L_{T}^{1}\left(L^{p}\right)}+\left\|\operatorname{div}\left[\Delta_{j}, \bar{\mu}\right] \nabla w\right\|_{L_{T}^{1}\left(L^{p}\right)}\right),
\end{aligned}
$$

and with $c=c_{p}$ in the definition of $e_{k}(t)$,

$$
\begin{aligned}
& \|u\|_{\widetilde{L}_{T}^{1}\left(\dot{B}_{p, 1}^{s-1}\right)}+\|u\|_{\widetilde{L}_{T}^{2}\left(\dot{B}_{p, 1}^{s}\right)} \leq \\
& \quad \leq C\left(\left\|u_{0}\right\|_{\dot{B}_{p, 1}^{s-1}(\omega)}+\|G\|_{\widetilde{L}_{T}^{1}\left(\dot{B}_{p, 1}^{s-1}(\omega)\right)}+\left\|\left(F_{1}, F_{2}\right)\right\|_{\widetilde{L}_{T}^{1}\left(\dot{B}_{p, 1}^{s-2}(\omega)\right)}\right) \\
& \quad(5.5) \quad+C \sum_{j} 2^{j(s-2)} \omega_{j}(T)\left(\left\|\operatorname{div}\left[\Delta_{j}, \bar{\nu}\right] \nabla d\right\|_{L_{T}^{1}\left(L^{p}\right)}+\left\|\operatorname{div}\left[\Delta_{j}, \bar{\mu}\right] \nabla w\right\|_{L_{T}^{1}\left(L^{p}\right)}\right) .
\end{aligned}
$$

First of all, we deal with the right hand side of (5.4). From Lemma 2.6 and 2.9 , we infer that

$$
\begin{aligned}
\left\|F_{1}\right\|_{\widetilde{L}_{T}^{1}\left(\dot{B}_{p, 1}^{s-2}\right)} & \leq C\left(\|\nabla \bar{\mu} \cdot \nabla u\|_{\widetilde{L}_{T}^{1}\left(\dot{B}_{p, 1}^{s-1}\right)}+\|\nabla(\bar{\lambda}+\bar{\mu}) d\|_{\widetilde{L}_{T}^{1}\left(\dot{B}_{p, 1}^{s-1}\right)}\right) \\
& \leq C\left(\|\bar{\mu}-\bar{\mu}(\underline{\rho})\|_{\widetilde{L}_{T}^{\infty}\left(\dot{B}_{p, 1}^{\frac{N}{p}}\right)}+\|\bar{\lambda}-\bar{\lambda}(\underline{\rho})\|_{\widetilde{L}_{T}^{\infty}\left(\dot{B}_{p, 1}^{\frac{N}{p}}\right)}\right)\|u\|_{\widetilde{L}_{T}^{1}\left(\dot{B}_{p, 1}^{s+1}\right)} \\
& \leq C A(T)\|\rho-\underline{\rho}\|_{\widetilde{L}_{T}^{\infty}\left(\dot{B}_{p, 1}^{\frac{N}{p}}\right)}\|u\|_{\widetilde{L}_{T}^{1}\left(\dot{B}_{p, 1}^{s+1}\right)} .
\end{aligned}
$$

Similarly, we have

$$
\left\|F_{2}\right\|_{\widetilde{L}_{T}^{1}\left(\dot{B}_{p, 1}^{s-2}\right)} \leq C A(T)\|\rho-\underline{\rho}\|_{\widetilde{L}_{T}^{\infty}\left(\dot{B}_{p, 1}^{\frac{N}{p}}\right)}\|u\|_{\widetilde{L}_{T}^{1}\left(\dot{B}_{p, 1}^{s+1}\right)} .
$$

While, we write

$$
\left[\Delta_{j}, \bar{\nu}\right] \nabla d=\left[\Delta_{j}, \bar{\nu}-\bar{\nu}(\underline{\rho})\right] \nabla d=\Delta_{j}((\bar{\nu}-\bar{\nu}(\underline{\rho})) \nabla d)-(\bar{\nu}-\bar{\nu}(\underline{\rho})) \Delta_{j} \nabla d,
$$

then by Lemma 2.6, Lemma 2.9 we get for $p \in[1, N]$

$$
\begin{gathered}
\sum_{j} 2^{j(s-2)}\left\|\operatorname{div} \Delta_{j}((\bar{\nu}-\bar{\nu}(\underline{\rho})) \nabla d)\right\|_{L_{T}^{1}\left(L^{p}\right)} \leq C A(T)\|\rho-\underline{\rho}\|_{\widetilde{L}_{T}^{\infty}\left(\dot{B}_{p, 1}^{\frac{N}{p}}\right)}\|u\|_{\widetilde{L}_{T}^{1}\left(\dot{B}_{p, 1}^{s+1}\right)}, \\
\sum_{j} 2^{j(s-2)}\left(\|\bar{\nu}-\bar{\nu}(\underline{\rho})\|_{L_{T}^{\infty}\left(L^{\infty}\right)}\left\|\Delta_{j} \operatorname{div} \nabla d\right\|_{L_{T}^{1}\left(L^{p}\right)}\right. \\
\left.\quad+\|\operatorname{div}(\bar{\nu}-\bar{\nu}(\underline{\rho}))\|_{L_{T}^{\infty}\left(L^{N}\right)}\left\|\Delta_{j} \nabla d\right\|_{L_{T}^{1}\left(L^{\frac{p N}{N-p}}\right)}\right) \leq \\
\leq C A(T)\|\rho-\underline{\rho}\|_{\widetilde{L}_{T}^{\infty}\left(\dot{B}_{p, 1}^{\frac{N}{p}}\right)}\|u\|_{\widetilde{L}_{T}^{1}\left(\dot{B}_{p, 1}^{s+1}\right)},
\end{gathered}
$$

which imply that

$$
\sum_{j} 2^{j(s-2)}\left\|\operatorname{div}\left[\Delta_{j}, \bar{\nu}\right] \nabla d\right\|_{L_{T}^{1}\left(L^{p}\right)} \leq C A(T)\|\rho-\underline{\rho}\|_{\widetilde{L}_{T}^{\infty}\left(\dot{B}_{p, 1}^{\frac{N}{p}}\right)}\|u\|_{\widetilde{L}_{T}^{1}\left(\dot{B}_{p, 1}^{s+1}\right)} .
$$


Similarly, we have

$$
\sum_{j} 2^{j(s-2)}\left\|\operatorname{div}\left[\Delta_{j}, \bar{\mu}\right] \nabla w\right\|_{L_{T}^{1}\left(L^{p}\right)} \leq C A(T)\|\rho-\underline{\rho}\|_{\widetilde{L}_{T}^{\infty}\left(\dot{B}_{p, 1}^{\frac{N}{p}}\right)}\|u\|_{\widetilde{L}_{T}^{1}\left(\dot{B}_{p, 1}^{s+1}\right)} .
$$

Then the first inequality of Proposition 5.1 (a) can be deduced from (5.4) and (5.6)-(5.9). On the other hand, using Lemma 2.6 and 2.9, we also have

$$
\left\|F_{1}\right\|_{\widetilde{L}_{T}^{1}\left(\dot{B}_{p, 1}^{s-2}\right)}+\left\|F_{2}\right\|_{\widetilde{L}_{T}^{1}\left(\dot{B}_{p, 1}^{s-2}\right)} \leq C A(T)\|\rho-\underline{\rho}\|_{\widetilde{L}_{T}^{\infty}\left(\dot{B}_{p, 1}^{\frac{N}{p}+1}\right)}\|u\|_{\widetilde{L}_{T}^{1}\left(\dot{B}_{p, 1}^{s}\right)},
$$

and by Lemma 2.8,

$$
\begin{gathered}
\sum_{j} 2^{j(s-2)}\left(\left\|\operatorname{div}\left[\Delta_{j}, \bar{\nu}\right] \nabla d\right\|_{L_{T}^{1}\left(L^{p}\right)}+\left\|\operatorname{div}\left[\Delta_{j}, \bar{\mu}\right] \nabla w\right\|_{L_{T}^{1}\left(L^{p}\right)}\right) \leq \\
\leq C A(T)\|\rho-\underline{\rho}\|_{\widetilde{L}_{T}^{\infty}\left(\dot{B}_{p, 1}^{\frac{N}{p}+1}\right)}\|u\|_{\widetilde{L}_{T}^{1}\left(\dot{B}_{p, 1}^{s}\right)},
\end{gathered}
$$

which together with (5.4) lead to the second inequality of Proposition 5.1 (a).

Next, we deal with the right hand side of (5.5). From Proposition 3.6 and 3.8 , it follows that

$$
\begin{gathered}
\left\|F_{1}\right\|_{\widetilde{L}_{T}^{1}\left(\dot{B}_{p, 1}^{s-2}(\omega)\right)} \leq C\left(\|\nabla \bar{\mu} \cdot \nabla u\|_{\widetilde{L}_{T}^{1}\left(\dot{B}_{p, 1}^{s-1}(\omega)\right)}+\|\nabla(\bar{\lambda}+\bar{\mu}) d\|_{\widetilde{L}_{T}^{1}\left(\dot{B}_{p, 1}^{s-1}(\omega)\right)}\right) \\
\quad \leq C\left(\|\bar{\mu}-\bar{\mu}(\underline{\rho})\|_{\widetilde{L}_{T}^{\infty}\left(\dot{B}_{p, 1}^{\frac{N}{p}}(\omega)\right)}+\|\bar{\lambda}-\bar{\lambda}(\underline{\rho})\|_{\widetilde{L}_{T}^{\infty}\left(\dot{B}_{p, 1}^{\frac{N}{p}}(\omega)\right)}\right)\|u\|_{\widetilde{L}_{T}^{1}\left(\dot{B}_{p, 1}^{s+1}\right)} \\
\quad \leq C A(T)\|\rho-\underline{\rho}\|_{\widetilde{L}_{T}^{\infty}\left(\dot{B}_{p, 1}^{\frac{N}{p}}(\omega)\right)}\|u\|_{\widetilde{L}_{T}^{1}\left(\dot{B}_{p, 1}^{s+1}\right)} .
\end{gathered}
$$

Similarly, we have

$$
\left\|F_{2}\right\|_{\widetilde{L}_{T}^{1}\left(\dot{B}_{p, 1}^{s-2}(\omega)\right)} \leq C A(T)\|\rho-\underline{\rho}\|_{\widetilde{L}_{T}^{\infty}\left(\dot{B}_{p, 1}^{\frac{N}{p}}(\omega)\right)}\|u\|_{\widetilde{L}_{T}^{1}\left(\dot{B}_{p, 1}^{s+1}\right)} .
$$

Notice that

$$
\left[\Delta_{j}, \bar{\nu}\right] \nabla d=\left[\Delta_{j}, \bar{\nu}-\bar{\nu}(\underline{\rho})\right] \nabla d, \quad\left[\Delta_{j}, \bar{\mu}\right] \nabla w=\left[\Delta_{j}, \bar{\mu}-\bar{\mu}(\underline{\rho})\right] \nabla w,
$$

which together with Lemma 5.2 and Proposition 3.8 ensures that

$$
\begin{aligned}
& \sum_{j} 2^{j(s-2)} \omega_{j}(T)\left(\left\|\operatorname{div}\left[\Delta_{j}, \bar{\nu}\right] \nabla d\right\|_{L_{T}^{1}\left(L^{p}\right)}+\left\|\operatorname{div}\left[\Delta_{j}, \bar{\mu}\right] \nabla w\right\|_{L_{T}^{1}\left(L^{p}\right)}\right) \leq \\
& \quad \leq C\left(\|\bar{\nu}-\bar{\nu}(\underline{\rho})\|_{\widetilde{L}_{T}^{\infty}\left(\dot{B}_{p, 1}^{\frac{N}{p}}(\omega)\right)}+\|\bar{\mu}-\bar{\mu}(\underline{\rho})\|_{\widetilde{L}_{T}^{\infty}\left(\dot{B}_{p, 1}^{\frac{N}{p}}(\omega)\right)}\right)\|u\|_{\widetilde{L}_{T}^{1}\left(\dot{B}_{p, 1}^{s+1}\right)} \\
& \quad \leq A(T)\|\rho-\underline{\rho}\|_{\widetilde{L}_{T}^{\infty}\left(\dot{B}_{p, 1}^{\frac{N}{p}}(\omega)\right)}\|u\|_{\widetilde{L}_{T}^{1}\left(\dot{B}_{p, 1}^{s+1}\right)} .
\end{aligned}
$$

Summing up (5.5) and (5.10)-(5.12), we obtain the inequality of Proposition 5.1 (b). 
Lemma 5.2 Let $p \in[1, N]$ and $s \in\left(-N \min \left(\frac{1}{p}, \frac{1}{p^{\prime}}\right), \frac{N}{p}\right]$. Assume that $f \in$ $\widetilde{L}_{T}^{\infty}\left(\dot{B}_{p, 1}^{\frac{N}{p}}(\omega)\right)$ and $g \in \widetilde{L}_{T}^{1}\left(\dot{B}_{p, 1}^{s+1}\right)$. Then there holds

$$
\sum_{j} 2^{j(s-1)} \omega_{j}(T)\left\|\operatorname{div}\left[\Delta_{j}, f\right] \nabla g\right\|_{L_{T}^{1}\left(L^{p}\right)} \leq C\|f\|_{\widetilde{L}_{T}^{\infty}\left(\dot{B}_{p, 1}^{\frac{N}{p}}(\omega)\right)}\|g\|_{\widetilde{L}_{T}^{1}\left(\dot{B}_{p, 1}^{s+1}\right)} .
$$

Proof. Using the Bony's decomposition, we write

$$
\begin{aligned}
{\left[f, \Delta_{j}\right] \partial_{k} g=} & {\left[T_{f}, \Delta_{j}\right] \partial_{k} g+T_{\partial_{k} \Delta_{j} g} f+R\left(f, \partial_{k} \Delta_{j} g\right) } \\
& -\Delta_{j}\left(T_{\partial_{k} g} f\right)-\Delta_{j} R\left(f, \partial_{k} g\right) .
\end{aligned}
$$

Using Lemma 3.5 (a) and (c) with $s_{1}=\frac{N}{p}$ and $s_{2}=s$, we get

$$
\begin{aligned}
\sum_{j} \omega_{j}(T) 2^{j(s-1)}\left\|\operatorname{div} \Delta_{j}\left(T_{\partial_{k} g} f\right)\right\|_{L_{T}^{1}\left(L^{p}\right)} & \leq C\|f\|_{\widetilde{L}_{T}^{\infty}\left(\dot{B}_{p, 1}^{\frac{N}{p}}(\omega)\right)}\|g\|_{\widetilde{L}_{T}^{1}\left(\dot{B}_{p, 1}^{s+1}\right)}, \\
\sum_{j} \omega_{j}(T) 2^{j(s-1)}\left\|\operatorname{div} \Delta_{j} R\left(f, \partial_{k} g\right)\right\|_{L_{T}^{1}\left(L^{p}\right)} & \leq C\|f\|_{\widetilde{L}_{T}^{\infty}\left(\dot{B}_{p, 1}^{\frac{N}{p}}(\omega)\right)}\|g\|_{\widetilde{L}_{T}^{1}\left(\dot{B}_{p, 1}^{s+1}\right)}
\end{aligned}
$$

Thanks to the proof of Lemma 4.3, we have

$$
T_{\partial_{k} \Delta_{j} g}^{\prime} f \triangleq T_{\partial_{k} \Delta_{j} g} f+R\left(f, \partial_{k} \Delta_{j} g\right)=\sum_{j^{\prime} \geq j-2} S_{j^{\prime}+2} \Delta_{j} \partial_{k} g \Delta_{j^{\prime}} f,
$$

then we get by Lemma 2.3 and (3.2) that

$$
\begin{aligned}
& \sum_{j} \omega_{j}(T) 2^{j(s-1)}\left\|\operatorname{div} T_{\partial_{k} \Delta_{j} g}^{\prime} f\right\|_{L_{T}^{1}\left(L^{p}\right)} \\
& \leq C \sum_{j} \omega_{j}(T) 2^{j s}\left(\left\|\Delta_{j} \nabla g\right\|_{L_{T}^{1}\left(L^{\infty}\right)} \sum_{j^{\prime} \geq j-2}\left\|\Delta_{j^{\prime}} f\right\|_{L_{T}^{1}\left(L^{p}\right)}\right. \\
& \left.\quad+\left\|\Delta_{j} g\right\|_{L_{T}^{1}\left(L^{\infty}\right)} \sum_{j^{\prime} \geq j-2} 2^{j^{\prime}}\left\|\Delta_{j^{\prime}} f\right\|_{L_{T}^{1}\left(L^{p}\right)}\right) \\
& \leq C \sum_{j} 2^{j\left(s+\frac{N}{p}\right)}\left\|\Delta_{j} g\right\|_{L_{T}^{1}\left(L^{p}\right)} \sum_{j^{\prime} \geq j-2}\left(2^{j}+2^{j^{\prime}}\right) \omega_{j^{\prime}}(T)\left\|\Delta_{j^{\prime}} f\right\|_{L_{T}^{1}\left(L^{p}\right)} \\
& \leq C\|f\|_{\widetilde{L}_{T}^{\infty}\left(\dot{B}_{p, 1}^{\frac{N}{p}}(\omega)\right)}\|g\|_{\widetilde{L}_{T}^{1}\left(\dot{B}_{p, 1}^{s+1}\right)}
\end{aligned}
$$

Set $h(x)=\left(\mathcal{F}^{-1} \varphi\right)(x)$. Thanks to the proof of Lemma 4.3, we have

$$
\begin{gathered}
{\left[T_{f}, \Delta_{j}\right] \partial_{k} g=\sum_{\left|j^{\prime}-j\right| \leq 4} 2^{(N+1) j} \int_{\mathbb{R}^{N}} \int_{0}^{1} y \cdot \nabla S_{j^{\prime}-1} f(x-\tau y) d \tau \partial_{k} h\left(2^{j} y\right) \Delta_{j^{\prime}} g(x-y) d y} \\
+2^{N j} \int_{\mathbb{R}^{N}} h\left(2^{j}(x-y)\right) \partial_{k} S_{j^{\prime}-1} f(y) \Delta_{j^{\prime}} g(y) d y
\end{gathered}
$$


from which and a similar argument of Lemma 3.5 (b), we infer that

$$
\sum_{j} \omega_{j}(T) 2^{j(s-1)}\left\|\operatorname{div}\left[T_{f}, \Delta_{j}\right] \partial_{k} g\right\|_{L_{T}^{1}\left(L^{p}\right)} \leq C\|f\|_{\widetilde{L}_{T}^{\infty}\left(\dot{B}_{p, 1}^{\frac{N}{p}}(\omega)\right)}\|g\|_{\widetilde{L}_{T}^{1}\left(\dot{B}_{p, 1}^{s+1}\right)} .
$$

Summing up the above estimates, we conclude the proof of Lemma 5.2.

To prove the uniqueness of the solution, we also need the following proposition.

Proposition 5.3 Let $p \in[2, N]$. Assume that $G \in L_{T}^{1}\left(\dot{B}_{p, \infty}^{-\frac{N}{p}}\right), u_{0} \in \dot{B}_{p, \infty}^{-\frac{N}{p}}$, and $\rho-\underline{\rho} \in L_{T}^{\infty}\left(\dot{B}_{p, 1}^{\frac{N}{p}}\right)$. Let $u$ be a solution of (5.1). Then there holds

$$
\begin{aligned}
& \|u\|_{\widetilde{L}_{T}^{1}\left(\dot{B}_{p, \infty}^{-\frac{N}{p}+2}\right)}+\|u\|_{\widetilde{L}_{T}^{2}\left(\dot{B}_{p, \infty}^{-\frac{N}{p}}+1\right.} \leq \\
& \quad \leq C\left(\left\|u_{0}\right\|_{\dot{B}_{p, \infty}^{-\frac{N}{p}}}+\|G(\tau)\|_{\widetilde{L}_{T}^{1}\left(\dot{B}_{p, \infty}^{-\frac{N}{p}}(\omega)\right)}+A(T)\|\rho-\underline{\rho}\|_{\widetilde{L}_{T}^{\infty}\left(\dot{B}_{p, 1}^{\frac{N}{p}}(\omega)\right)}\|u\|_{\widetilde{L}_{T}^{1}\left(\dot{B}_{p, \infty}^{-\frac{N}{p}+2}\right)}\right) .
\end{aligned}
$$

Proof. We closely follow the proof of Proposition 5.1. From (5.3), we infer that

$$
\begin{aligned}
& \|u\|_{\widetilde{L}_{T}^{1}\left(\dot{B}_{p, \infty}^{-\frac{N}{p}+2}\right)}+\|u\|_{\widetilde{L}_{T}^{2}\left(\dot{B}_{p, \infty}^{-\frac{N}{p}+1}\right)} \leq \\
& \leq C\left(\left\|u_{0}\right\|_{\dot{B}_{p, \infty}^{-\frac{N}{p}}(\omega)}+\|G\|_{\widetilde{L}_{T}^{1}\left(\dot{B}_{p, \infty}^{-\frac{N}{p}}(\omega)\right)}+\left\|\left(F_{1}, F_{2}\right)\right\|_{\widetilde{L}_{T}^{1}\left(\dot{B}_{p, \infty}^{-\frac{N}{p}-1}(\omega)\right)}\right) \\
& \quad+C \sup _{j \in \mathbb{Z}} 2^{j\left(-\frac{N}{p}-1\right)} \omega_{j}(T)\left(\left\|\operatorname{div}\left[\Delta_{j}, \bar{\nu}\right] \nabla d\right\|_{L_{T}^{1}\left(L^{p}\right)}+\left\|\operatorname{div}\left[\Delta_{j}, \bar{\mu}\right] \nabla w\right\|_{L_{T}^{1}\left(L^{p}\right)}\right) .
\end{aligned}
$$

We use Proposition 3.7 to get

$$
\left\|\left(F_{1}, F_{2}\right)\right\|_{\widetilde{L}_{T}^{1}\left(\dot{B}_{p, \infty}^{-\frac{N}{p}-1}(\omega)\right)} \leq C A(T)\|\rho-\underline{\rho}\|_{\widetilde{L}_{T}^{\infty}\left(\dot{B}_{p, 1}^{\frac{N}{p}}(\omega)\right)}\|u\|_{\widetilde{L}_{T}^{1}\left(\dot{B}_{p, \infty}^{-\frac{N}{p}}+2\right)} .
$$

From Lemma 5.4, the second term on the right hand side of (5.13) is bounded by

$$
C A(T)\|\rho-\underline{\rho}\|_{\widetilde{L}_{T}^{\infty}\left(\dot{B}_{p, 1}^{\frac{N}{p}}(\omega)\right)}\|u\|_{\widetilde{L}_{T}^{1}\left(\dot{B}_{p, \infty}^{-\frac{N}{p}+2}\right)} .
$$

This completes the proof of Proposition 5.3.

Lemma 5.4 Let $p \in[1, N]$. Assume that $f \in \widetilde{L}_{T}^{\infty}\left(\dot{B}_{p, 1}^{\frac{N}{p}}(\omega)\right)$ and $g \in \widetilde{L}_{T}^{1}\left(\dot{B}_{p, \infty}^{-\frac{N}{p}+1}\right)$. Then there holds

$$
\sup _{j \in \mathbb{Z}} 2^{j\left(-\frac{N}{p}-1\right)} \omega_{j}(T)\left\|\operatorname{div}\left[\Delta_{j}, f\right] \nabla g\right\|_{L_{T}^{1}\left(L^{p}\right)} \leq C\|f\|_{\widetilde{L}_{T}^{\infty}\left(\dot{B}_{p, 1}^{\frac{N}{p}}(\omega)\right)}\|g\|_{\widetilde{L}_{T}^{1}\left(\dot{B}_{p}^{-\frac{N}{p}+1}\right)} .
$$

The proof of Lemma 5.4 is very similar to that of Lemma 5.2. Here we omit its proof. 


\section{The proof of existence}

We set

$$
a(t, x)=\frac{\rho(t, x)-\bar{\rho}_{0}}{\bar{\rho}_{0}}, \quad \bar{\mu}(\rho)=\frac{\mu(\rho)}{\rho}, \quad \bar{\lambda}(\rho)=\frac{\lambda(\rho)}{\rho} .
$$

Then the system (1.1) reads

$$
\left\{\begin{array}{l}
\partial_{t} a+u \cdot \nabla a=F \\
\partial_{t} u-\operatorname{div}(\bar{\mu} \nabla u)-\nabla((\bar{\lambda}+\bar{\mu}) \operatorname{div} u)=G, \\
\left.(a, u)\right|_{t=0}=\left(a_{0}, u_{0}\right),
\end{array}\right.
$$

with $a_{0}=\frac{\rho_{0}(x)-\bar{\rho}_{0}}{\bar{\rho}_{0}}$ and

$$
\begin{aligned}
& F(a, u)=-(1+a) \operatorname{div} u, \\
& G(a, u)=-u \cdot \nabla u+\frac{\bar{\rho}_{0} P^{\prime}(\rho)}{\rho} \nabla a+\frac{\mu(\rho)}{\rho^{2}} \nabla \rho \cdot \nabla u+\frac{\mu(\rho)+\lambda(\rho)}{\rho^{2}} \nabla \rho \operatorname{div} u .
\end{aligned}
$$

Step 1. The approximate solution sequence.

We smooth out the data as follows:

$$
a_{0}^{n}=S_{n+K} a_{0}, \quad u_{0}^{n}=S_{n} u_{0},
$$

where $K \in \mathbb{Z}$ is chosen such that

$$
\bar{\rho}_{0}\left(1+a_{0}^{n}(x)\right) \geq \frac{3}{4} c_{0} .
$$

A standard linearized argument (as in the proof of Theorem 4.2 in [12]) will ensure that the system (6.1) with the smooth data $\left(a_{0}^{n}, u_{0}^{n}\right)$ has a solution $\left(a^{n}, u^{n}\right)$ on a time interval $\left[0, T_{n}\right]$ for some $T_{n}>0$ such that

$$
\left\{\begin{array}{l}
a^{n} \in C\left(\left[0, T_{n}\right] ; \dot{B}_{p, 1}^{\frac{N}{p}} \cap \dot{B}_{p, 1}^{\frac{N}{p}+1}\right) \text { and } \\
u^{n} \in C\left(\left[0, T_{n}\right] ; \dot{B}_{p, 1}^{\frac{N}{p}-1} \cap \dot{B}_{p, 1}^{\frac{N}{p}}\right) \cap L^{1}\left(\left[0, T_{n}\right] ; \dot{B}_{p, 1}^{\frac{N}{p}+1} \cap \dot{B}_{p, 1}^{\frac{N}{p}+2}\right) .
\end{array}\right.
$$

In what follows, we also denote by $T_{n}$ the maximal lifespan of the solution $\left(a^{n}, u^{n}\right)$.

Step 2. Uniform estimates.

Let

$$
E_{0}:=\left\|a_{0}\right\|_{\dot{B}_{p, 1}^{\frac{N}{p}}}+\left\|u_{0}\right\|_{\dot{B}_{p, 1}^{\frac{N}{p}}-1}
$$

and $T \in\left(0, T_{n}\right)$. We assume that the solutions $\left(a^{n}, u^{n}\right)$ satisfy the following inequalities for some positive constants $c_{1}, C_{0}, A_{0}$ and $\eta$ (to be determined later): 
(H1) $\bar{\rho}_{0}\left(1+a_{0}^{n}(t, x)\right) \geq \frac{c_{0}}{2}$ for any $(t, x) \in[0, T] \times \mathbb{R}^{N}$;

(H2) $\bar{\mu}^{n}(t, x) \geq c_{1}, \bar{\lambda}^{n}(t, x)+2 \bar{\mu}^{n}(t, x) \geq c_{1}$ for any $(t, x) \in[0, T] \times \mathbb{R}^{N}$;

(H3) $\left\|a^{n}\right\|_{\widetilde{L}_{T}^{\infty}\left(\dot{B}_{p, 1}^{\frac{N}{p}}\right)}+\left\|u^{n}\right\|_{\widetilde{L}_{T}^{\infty}\left(\dot{B}_{p, 1}^{\frac{N}{p}-1}\right)} \leq C_{0} E_{0}$

(H4) $\left\|a^{n}\right\|_{\widetilde{L}_{T}^{\infty}\left(\dot{B}_{p, 1}^{\frac{N}{p}}(\omega)\right)} \leq A_{0} \eta,\left\|u^{n}\right\|_{\widetilde{L}_{T}^{1}\left(\dot{B}_{p, 1}^{\frac{N}{p}+1}\right)}+\left\|u^{n}\right\|_{\widetilde{L}_{T}^{2}\left(\dot{B}_{p, 1}^{\frac{N}{p}}\right)} \leq \eta$.

In what follows, we will show that if the conditions (H1) to (H4) are satisfied for some $T>0$, then they are actually satisfied with strict inequalities. Since all those conditions depend continuously on the time variable and are satisfied initially, a standard bootstrap argument will ensure that (H1) to (H4) are indeed satisfied for $T$.

First of all, we get by Proposition 4.1 that

$$
\left\|a^{n}\right\|_{\widetilde{L}_{T}^{\infty}\left(\dot{B}_{p, 1}^{\frac{N}{p}}\right)} \leq e^{C V^{n}(T)}\left(\left\|a_{0}\right\|_{\dot{B}_{p, 1}^{\frac{N}{p}}}+\left\|F^{n}\right\|_{\widetilde{L}_{T}^{1}\left(\dot{B}_{p, 1}^{\frac{N}{p}}\right)}\right),
$$

and by Proposition 5.1, we have

$$
\begin{aligned}
\left\|u^{n}\right\|_{\widetilde{L}_{T}^{\infty}\left(\dot{B}_{p, 1}^{\frac{N}{p}-1}\right)} \leq & C\left\|u_{0}\right\|_{\dot{B}_{p, 1}^{\frac{N}{p}-1}}+C\left\|G^{n}\right\|_{\widetilde{L}_{T}^{1}\left(\dot{B}_{p, 1}^{\frac{N}{p}-1}\right)} \\
& +C A^{n}(T)\left\|a^{n}\right\|_{\widetilde{L}_{T}^{\infty}\left(\dot{B}_{p, 1}^{\frac{N}{p}}\right)}\left\|u^{n}\right\|_{\widetilde{L}_{T}^{1}\left(\dot{B}_{p, 1}^{\frac{N}{p}+1}\right)}
\end{aligned}
$$

where

$$
V^{n}(t)=\int_{0}^{t}\left\|\nabla u^{n}(\tau)\right\|_{\dot{B}_{p, 1}^{\frac{N}{p}}} d \tau \quad \text { and } \quad A^{n}(T)=\left(1+\left\|a^{n}\right\|_{\widetilde{L}_{T}^{\infty}\left(\dot{B}_{p, 1}^{\frac{N}{p}}\right)}\right)^{\left[\frac{N}{p}\right]+3} .
$$

For $F^{n}$, we apply Lemma 2.6 to get

$$
\left\|F^{n}\right\|_{\widetilde{L}_{T}^{1}\left(\dot{B}_{p, 1}^{\frac{N}{p}}\right)} \leq\left\|u^{n}\right\|_{\widetilde{L}_{T}^{1}\left(\dot{B}_{p, 1}^{\frac{N}{p}+1}\right)}+C\left\|a^{n}\right\|_{\widetilde{L}_{T}^{\infty}\left(\dot{B}_{p, 1}^{\frac{N}{p}}\right)}\left\|u^{n}\right\|_{\widetilde{L}_{T}^{1}\left(\dot{B}_{p, 1}^{\frac{N}{p}+1}\right)},
$$

and for $G^{n}$, we use Lemma 2.6 and 2.9 to get

$$
\begin{aligned}
\left\|G^{n}\right\|_{\widetilde{L}_{T}^{1}\left(\dot{B}_{p, 1}^{\frac{N}{p}-1}\right)} \leq & C\left\|u^{n}\right\|_{\widetilde{L}_{T}^{\infty}\left(\dot{B}_{p, 1}^{\frac{N}{p}-1}\right)}\left\|u^{n}\right\|_{\widetilde{L}_{T}^{1}\left(\dot{B}_{p, 1}^{\frac{N}{p}+1}\right)} \\
& +C A^{n}(T)\left\|a^{n}\right\|_{\widetilde{L}_{T}^{\infty}\left(\dot{B}_{p, 1}^{\frac{N}{p}}\right)}\left(T+\left\|u^{n}\right\|_{\widetilde{L}_{T}^{1}\left(\dot{B}_{p, 1}^{\frac{N}{p}+1}\right)}\right) .
\end{aligned}
$$

Plugging the above two estimates into (6.4) and (6.5), we obtain

$$
\begin{aligned}
& \left\|a^{n}\right\|_{\widetilde{L}_{T}^{\infty}\left(\dot{B}_{p, 1}^{\frac{N}{p}}\right)}+\left\|u^{n}\right\|_{\widetilde{L}_{T}^{\infty}\left(\dot{B}_{p, 1}^{\frac{N}{p}-1}\right)} \leq \\
& \quad \leq C_{1} e^{C_{1} V^{n}(T)}\left(E_{0}+\left(C_{0} E_{0}+1\right) \eta\right)+C_{1} A^{n}(T) C_{0} E_{0}(T+\eta) .
\end{aligned}
$$


Next, we get by Proposition 4.2 that

$$
\left\|a^{n}\right\|_{\widetilde{L}_{T}^{\infty}\left(\dot{B}_{p, 1}^{\frac{N}{p}}(\omega)\right)} \leq e^{C V^{n}(T)}\left(\left\|a_{0}\right\|_{\dot{B}_{p, 1}^{\frac{N}{p}(\omega)}}+\left\|F^{n}\right\|_{\widetilde{L}_{T}^{1} \dot{B}_{p, 1}^{\frac{N}{p}}(\omega)}\right),
$$

and by Proposition 5.1, we have

$$
\begin{aligned}
& \left\|u^{n}\right\|_{\widetilde{L}_{T}^{1}\left(\dot{B}_{p, 1}^{\frac{N}{p}+1}\right)}+\left\|u^{n}\right\|_{\widetilde{L}_{T}^{2}\left(\dot{B}_{p, 1}^{\frac{N}{p}}\right)} \leq
\end{aligned}
$$

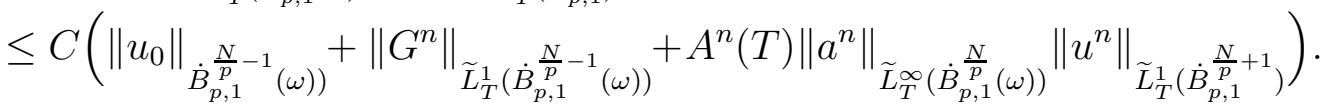

For $F^{n}$, we have

$$
\left\|F^{n}\right\|_{\widetilde{L}_{T}^{1}\left(\dot{B}_{p, 1}^{\frac{N}{p}}(\omega)\right)} \leq 2\left\|F^{n}\right\|_{\widetilde{L}_{T}^{1}\left(\dot{B}_{p, 1}^{\frac{N}{p}}\right)} \leq C\left(1+\left\|a^{n}\right\|_{\widetilde{L}_{T}^{\infty}\left(\dot{B}_{p, 1}^{\frac{N}{p}}\right)}\left\|u^{n}\right\|_{\widetilde{L}_{T}^{1}\left(\dot{B}_{p, 1}^{\frac{N}{p}+1}\right)},\right.
$$

and for $G^{n}$, we use Proposition 3.6 and 3.8 to get

$$
\left\|G^{n}\right\|_{\widetilde{L}_{T}^{1}\left(\dot{B}_{p, 1}^{\frac{N}{p}-1}(\omega)\right)} \leq C\left\|u^{n}\right\|_{\widetilde{L}_{T}^{2}\left(\dot{B}_{p, 1}^{\frac{N}{p}}\right)}^{2}+C A^{n}(T)\left\|a^{n}\right\|_{\widetilde{L}_{T}^{\infty}\left(\dot{B}_{p, 1}^{\frac{N}{p}}(\omega)\right)}\left(T+\left\|u^{n}\right\|_{\widetilde{L}_{T}^{1}\left(\dot{B}_{p, 1}^{\frac{N}{p}}+1\right.}\right) .
$$

Plugging the above two estimates into (6.7) and (6.8), we obtain

$$
\begin{aligned}
\left\|a^{n}\right\|_{\widetilde{L}_{T}^{\infty}\left(\dot{B}_{p, 1}^{\frac{N}{p}}(\omega)\right)} & \leq e^{C_{1} V^{n}(T)}\left(\left\|a_{0}\right\|_{\dot{B}_{p, 1}^{\frac{N}{p}}(\omega)}+C_{2}\left(1+C_{0} E_{0}\right) \eta\right), \\
\left\|u^{n}\right\|_{\widetilde{L}_{T}^{1}\left(\dot{B}_{p, 1}^{\frac{N}{p}+1}\right)} & +\left\|u^{n}\right\|_{\widetilde{L}_{T}^{2}\left(\dot{B}_{p, 1}^{\frac{N}{p}}\right)} \leq \\
& \leq C_{3}\left(\left\|u_{0}\right\|_{\left.\dot{B}_{p, 1}^{\frac{N}{p}-1}(\omega)\right)}+\eta^{2}+A_{0} A^{n}(T) \eta(T+\eta)\right) .
\end{aligned}
$$

According to the definition of $V^{n}$ and $A^{n}$, we have

$$
V^{n}(T) \leq \eta, \quad A^{n}(T) \leq\left(1+C_{0} E_{0}\right)^{\left[\frac{N}{p}\right]+3} .
$$

Let $C_{0}=4 C_{1}$ and $A_{0}=2 C_{2}\left(1+C_{0} E_{0}\right)$. Then we take $\eta$ small enough such that

$$
\left\{\begin{array}{l}
e^{C_{1} \eta}<\frac{3}{2},\left(C_{0} E_{0}+1\right) \eta \leq E_{0}, C_{1}\left(C_{0} E_{0}+1\right)^{\left[\frac{N}{p}\right]+3} \eta \leq \frac{1}{16} \\
C_{3} \eta \leq \frac{1}{6}, C_{3} A_{0}\left(1+C_{0} E_{0}\right)^{\left[\frac{N}{p}\right]+3} \eta \leq \frac{1}{6}
\end{array}\right.
$$

Next, we take $T$ small enough such that

$$
C_{1}\left(C_{0} E_{0}+1\right)^{\left[\frac{N}{p}\right]+3} T \leq \frac{1}{16}, C_{3} A_{0}\left(1+C_{0} E_{0}\right)^{\left[\frac{N}{p}\right]+3} T \leq \frac{1}{6} .
$$

and note that $\omega_{k}(0)=0$ and $\left(a_{0}, u_{0}\right) \in \dot{B}_{p, 1}^{\frac{N}{p}} \times \dot{B}_{p, 1}^{\frac{N}{p}-1}$, we can also take $T$ small enough such that

$$
\left\|a_{0}\right\|_{\dot{B}_{p, 1}^{\frac{N}{p}}(\omega)} \leq \frac{A_{0}}{12} \eta, \quad\left\|u_{0}\right\|_{\dot{B}_{p, 1}^{\frac{N}{p}-1}(\omega)} \leq \frac{\eta}{6 C_{3}}
$$


Then it follows from (6.6) that

$$
\left\|a^{n}\right\|_{\widetilde{L}_{T}^{\infty}\left(\dot{B}_{p, 1}^{\frac{N}{p}}\right)}+\left\|u^{n}\right\|_{\widetilde{L}_{T}^{\infty}\left(\dot{B}_{p, 1}^{\frac{N}{p}-1}\right)} \leq \frac{7}{8} C_{0} E_{0}
$$

and from (6.9) and (6.10), we infer that

$$
\left\|a^{n}\right\|_{\widetilde{L}_{T}^{\infty}\left(\dot{B}_{p, 1}^{\frac{N}{p}}(\omega)\right)} \leq \frac{7}{8} A_{0} \eta,\left\|u^{n}\right\|_{\widetilde{L}_{T}^{1}\left(\dot{B}_{p, 1}^{\frac{N}{p}+1}\right)}+\left\|u^{n}\right\|_{\widetilde{L}_{T}^{2}\left(\dot{B}_{p, 1}^{\frac{N}{p}}\right)} \leq \frac{2}{3} \eta,
$$

which ensure that (H3) and (H4) are satisfied with strict inequalities for $T$ and $\eta$ satisfying (6.11)-(6.13).

Let $X_{n}(t, x)$ be a solution of

$$
\frac{d}{d t} X_{n}(t, x)=u^{n}\left(t, X_{n}(t, x)\right), \quad X_{n}(0, x)=x,
$$

and we denote by $X_{n}^{-1}(t, x)$ the inverse of $X_{n}(t, x)$. Then $a^{n}(t, x)$ can be solved as

$$
a^{n}(t, x)=a_{0}^{n}\left(X_{n}^{-1}(t, x)\right)+\int_{0}^{t} F^{n}\left(\tau, X_{n}\left(\tau, X_{n}^{-1}(t, x)\right)\right) d \tau,
$$

thus, we have

$$
\bar{\rho}_{0}\left(1+a^{n}(t, x)\right)=\rho_{0}^{n}\left(X_{n}^{-1}(t, x)\right)+\bar{\rho}_{0} \int_{0}^{t} F^{n}\left(\tau, X_{n}\left(\tau, X_{n}^{-1}(t, x)\right)\right) d \tau .
$$

On the other hand, we have

$$
\left\|F^{n}\right\|_{L_{T}^{1}\left(L^{\infty}\right)} \leq\|\nabla u\|_{L_{T}^{1}\left(L^{\infty}\right)}\left(1+\|a\|_{L_{T}^{\infty}\left(L^{\infty}\right)}\right) \leq C_{4}\left(1+C_{0} E_{0}\right) \eta .
$$

We take $\eta$ such that

$$
C_{4}\left(1+C_{0} E_{0}\right) \eta<\frac{1}{8} c_{0} .
$$

Then from (6.14) and (6.2), it follows that

$$
\bar{\rho}_{0}\left(1+a^{n}(t, x)\right) \geq \frac{3}{4} c_{0}-\frac{1}{8} c_{0} \geq \frac{5}{8} c_{0},
$$

that is, (H1) is satisfied with the strict inequality. Finally, take

$$
c_{1}=\frac{1}{2} \min \left(\inf _{|\rho| \leq \bar{\rho}_{0}\left(1+C_{0} E_{0}\right)} \bar{\mu}(\rho), \inf _{|\rho| \leq \bar{\rho}_{0}\left(1+C_{0} E_{0}\right)}(\bar{\lambda}(\rho)+2 \bar{\mu}(\rho))\right),
$$

which ensures that (H2) is satisfied with strict inequality. 
Let $T^{*}$ be the supremum of all time $T$ such that (6.12) and (6.13) are satisfied. We need to prove that $T_{n} \geq T^{*}$. If $T_{n}<T^{*}$, then we can prove that

$$
\left\{\begin{array}{l}
a^{n} \in \widetilde{L}^{\infty}\left(0, T_{n} ; \dot{B}_{p, 1}^{\frac{N}{p}} \cap \dot{B}_{p, 1}^{\frac{N}{p}+1}\right) \quad \text { and } \\
u^{n} \in \widetilde{L}^{\infty}\left(0, T_{n} ; \dot{B}_{p, 1}^{\frac{N}{p}-1} \cap \dot{B}_{p, 1}^{\frac{N}{p}}\right) \cap L^{1}\left(\left[0, T_{n}\right] ; \dot{B}_{p, 1}^{\frac{N}{p}+1} \cap \dot{B}_{p, 1}^{\frac{N}{p}+2}\right),
\end{array}\right.
$$

thus, the solution $\left(a^{n}, u^{n}\right)$ can be continued beyond $T^{*}$. Indeed, from Proposition 4.1, we have

$$
\left\|a^{n}\right\|_{\widetilde{L}_{t}^{\infty}\left(\dot{B}_{p, 1}^{\frac{N}{p}+1}\right)} \leq e^{C V^{n}(t)}\left(\left\|a_{0}^{n}\right\|_{\dot{B}_{p, 1}^{\frac{N}{p}+1}}+\int_{0}^{t}\left\|F^{n}(\tau)\right\|_{\dot{B}_{p, 1}^{\frac{N}{p}+1}} d \tau\right),
$$

and by Proposition 5.1 (a), we have

$$
\begin{aligned}
& \left\|u^{n}\right\|_{\widetilde{L}_{T}^{\infty}\left(\dot{B}_{p, 1}^{\frac{N}{p}}\right)}+\left\|u^{n}\right\|_{\widetilde{L}_{T}^{1}\left(\dot{B}_{p, 1}^{\frac{N}{p}+2}\right)} \leq C\left\|u_{0}^{n}\right\|_{\dot{B}_{p, 1}^{\frac{N}{p}}}+C\left\|G^{n}\right\|_{\widetilde{L}_{T}^{1}\left(\dot{B}_{p, 1}^{\frac{N}{p}}\right)} \\
& +C A^{n}(T)\left\|a^{n}\right\|_{\widetilde{L}_{T}^{\infty}\left(\dot{B}_{p, 1}^{\frac{N}{p}+1}\right)}\left\|u^{n}\right\|_{\widetilde{L}_{T}^{1}\left(\dot{B}_{p, 1}^{\frac{N}{p}+1}\right)},
\end{aligned}
$$

On the other hand, we use Lemma 2.5 and the embedding $\dot{B}_{p, 1}^{\frac{N}{p}} \hookrightarrow L^{\infty}$ to get

$$
\left\|F^{n}\right\|_{\dot{B}_{p, 1}^{\frac{N}{p}+1}} \leq\left\|u^{n}\right\|_{\dot{B}_{p, 1}^{\frac{N}{p}+2}}+C\left\|a^{n}\right\|_{\dot{B}_{p, 1}^{\frac{N}{p}}}\left\|u^{n}\right\|_{\dot{B}_{p, 1}^{\frac{N}{p}+2}}+C\left\|a^{n}\right\|_{\dot{B}_{p, 1}^{\frac{N}{p}+1}}\left\|u^{n}\right\|_{\dot{B}_{p, 1}^{\frac{N}{p}+1}},
$$

and by Lemma 2.6 and 2.9 , we have

$$
\begin{aligned}
\left\|G^{n}\right\|_{\widetilde{L}_{T}^{1}\left(\dot{B}_{p, 1}^{\frac{N}{p}}\right)} \leq & C\left\|u^{n}\right\|_{\widetilde{L}_{T}^{\infty} \dot{B}_{p, 1}^{\frac{N}{p}}}\left\|u^{n}\right\|_{\widetilde{L}_{T}^{1} \dot{B}_{p, 1}^{\frac{N}{p}+1}} \\
& +C A^{n}(T)\left\|a^{n}\right\|_{\widetilde{L}_{T}^{\infty} \dot{B}_{p, 1}^{\frac{N}{p}+1}}\left(T+\left\|u^{n}\right\|_{\widetilde{L}_{T}^{1} \dot{B}_{p, 1}^{\frac{N}{p}+1}}\right),
\end{aligned}
$$

which together with (6.16), (6.17) and (H3-H4) implies (6.15).

Step 3. Existence of a solution.

We will use a compact argument to prove that the approximate sequence $\left\{a^{n}, u^{n}\right\}_{n \in \mathbb{N}}$ tends to some function $(a, u)$ which satisfies the system (6.1) in the sense of distribution.

Since $\left\{u^{n}\right\}$ is uniformly bounded in $L_{T}^{1}\left(\dot{B}_{p, 1}^{\frac{N}{p}+1}\right) \cap L_{T}^{\infty}\left(\dot{B}_{p, 1}^{\frac{N}{p}-1}\right)$, we get by the interpolation that $\left\{u^{n}\right\}_{n \in \mathbb{N}}$ is also uniformly bounded in $L_{T}^{q}\left(\dot{B}_{p, 1}^{\frac{N}{p}-1+2 / q}\right)$ for any $q \in[1, \infty]$. By Lemma 2.6, we have

$$
\begin{aligned}
& \left\|a^{n} \operatorname{div} u^{n}\right\|_{\dot{B}_{p, 1}^{\frac{N}{p}-1}} \leq C\left\|a^{n}\right\|_{\dot{B}_{p, 1}^{\frac{N}{p}}}\left\|u^{n}\right\|_{\dot{B}_{p, 1}^{\frac{N}{p}}}, \\
& \left\|u^{n} \cdot \nabla a^{n}\right\|_{\dot{B}_{p, 1}^{\frac{N}{p}-1}} \leq C\left\|a^{n}\right\|_{\dot{B}_{p, 1}^{\frac{N}{p}}}^{\frac{N}{p}}\left\|u^{n}\right\|_{\dot{B}_{p, 1}^{\frac{N}{p}}},
\end{aligned}
$$


from which and the first equation of the system (6.1), we infer that $\left\{\partial_{t} a^{n}\right\}_{n \in \mathbb{N}}$ is uniformly bounded in $L_{T}^{2}\left(\dot{B}_{p, 1}^{\frac{N}{p}-1}\right)$. On the other hand, by Lemma 2.6 and Lemma 2.9, we have

$$
\begin{gathered}
\left\|u^{n} \cdot \nabla u^{n}\right\|_{\dot{B}_{p, 1}^{\frac{N}{p}-3 / 2}} \leq C\left\|u^{n}\right\|_{\dot{B}_{p, 1}^{\frac{N}{p}-1}}\left\|u^{n}\right\|_{\dot{B}_{p, 1}^{\frac{N}{p}+1 / 2}}, \\
\left\|\frac{\bar{\rho}_{0} P^{\prime}\left(\rho^{n}\right)}{\rho^{n}} \nabla a^{n}\right\|_{\dot{B}_{p, 1}^{\frac{N}{p}-1}} \leq C\left(\left\|a^{n}\right\|_{\infty}\right)\left\|a^{n}\right\|_{\dot{B}_{p, 1}^{\frac{N}{p}}}\left(1+\left\|a^{n}\right\|_{\dot{B}_{p, 1}^{\frac{N}{p}}}\right),
\end{gathered}
$$

and

$$
\begin{aligned}
& \left\|\operatorname{div}\left(\bar{\mu}^{n} \nabla u^{n}\right)\right\|_{\dot{B}_{p, 1}^{\frac{N}{p}-3 / 2}}+\left\|\nabla\left(\left(\bar{\lambda}^{n}+\bar{\mu}^{n}\right) \operatorname{div} u^{n}\right)\right\|_{\dot{B}_{p, 1}^{\frac{N}{p}-3 / 2}}+\left\|G_{1}^{n}\right\|_{\dot{B}_{p, 1}^{\frac{N}{p}-3 / 2}} \leq \\
& \quad \leq C\left(\left\|a^{n}\right\|_{\infty}\right)\left\|a^{n}\right\|_{\dot{B}_{p, 1}^{\frac{N}{p}}}\left(1+\left\|a^{n}\right\|_{\dot{B}_{p, 1}^{\frac{N}{p}}}\right)\left\|u^{n}\right\|_{\dot{B}_{p, 1}^{\frac{N}{p}+1 / 2}},
\end{aligned}
$$

where

$$
G_{1}^{n} \stackrel{\text { def }}{=} \frac{\mu\left(\rho^{n}\right)}{\left(\rho^{n}\right)^{2}} \nabla \rho^{n} \cdot \nabla u^{n}+\frac{\mu\left(\rho^{n}\right)+\lambda\left(\rho^{n}\right)}{\left(\rho^{n}\right)^{2}} \nabla \rho^{n} \operatorname{div} u^{n} .
$$

Then, from the second equation of the system (6.1), we infer that $\left\{\partial_{t} u^{n}\right\}_{n \in \mathbb{N}}$ is uniformly bounded in $L_{T}^{\frac{4}{3}}\left(\dot{B}_{p, 1}^{\frac{N}{p}-\frac{3}{2}}+\dot{B}_{p, 1}^{\frac{N}{p}-1}\right)$.

Let $\left\{\chi_{j}\right\}_{j \in \mathbb{N}}$ be a sequence of smooth functions supported in the ball $B(0, j+1)$ and equal to 1 on $B(0, j)$. The above proof ensures that for any $j \in \mathbb{N},\left\{\chi_{j} a^{n}\right\}_{n \in \mathbb{N}}$ is uniformly bounded in $C^{\frac{1}{2}}\left([0, T] ; \dot{B}_{p, 1}^{\frac{N}{p}-1}\right)$, and $\left\{\chi_{j} u^{n}\right\}_{n \in \mathbb{N}}$ is uniformly bounded in $C^{\frac{1}{4}}\left([0, T] ; \dot{B}_{p, 1}^{\frac{N}{p}-\frac{3}{2}}+\dot{B}_{p, 1}^{\frac{N}{p}-1}\right)$. Since the embeddings

$$
\dot{B}_{p, 1}^{\frac{N}{p}-1} \cap \dot{B}_{p, 1}^{\frac{N}{p}} \hookrightarrow \dot{B}_{p, 1}^{\frac{N}{p}-1} \quad \text { and } \quad \dot{B}_{p, 1}^{\frac{N}{p}-3 / 2} \cap \dot{B}_{p, 1}^{\frac{N}{p}-1} \hookrightarrow \dot{B}_{p, 1}^{\frac{N}{p}-3 / 2}
$$

are locally compact, by applying Ascoli's theorem and Cantor's diagonal process, there exists some function $(a, u)$ such that for any $j \in \mathbb{N}$,

$$
\left\{\begin{array}{lll}
\chi_{j} a^{n} \longrightarrow \chi_{j} a & \text { in } \quad C\left([0, T] ; \dot{B}_{p_{p}^{1}}^{\frac{N}{p}-1}\right) \\
\chi_{j} u^{n} \longrightarrow \chi_{j} u & \text { in } \quad C\left([0, T] ; \dot{B}_{p, 1}^{\frac{N}{p}-\frac{3}{2}}\right)
\end{array}\right.
$$

as $n$ tends to $\infty$ (up to a subsequence).

By interpolation, we also have

$$
\left\{\begin{array}{llll}
\chi_{j} a^{n} \longrightarrow \chi_{j} a & \text { in } \quad C\left([0, T] ; \dot{B}_{p, 1}^{\frac{N}{p}-s}\right), & \forall 0<s \leq 1 \\
\chi_{j} u^{n} \longrightarrow \chi_{j} u & \text { in } \quad L^{1}\left([0, T] ; \dot{B}_{p, 1}^{\frac{N}{p}+s}\right), & \forall-\frac{3}{2} \leq s<1 .
\end{array}\right.
$$


Furthermore, we actually have

$$
\left\{\begin{array}{l}
(a, u) \in \widetilde{L}_{T}^{\infty}\left(\dot{B}_{p, 1}^{\frac{N}{p}}\right) \otimes\left(\widetilde{L}_{T}^{\infty}\left(\dot{B}_{p, 1}^{\frac{N}{p}-1}\right) \cap L^{1}\left(0, T ; \dot{B}_{p, 1}^{\frac{N}{p}+1}\right)\right), \\
\bar{\rho}_{0}(1+a(t, x)) \geq \frac{c_{0}}{2} .
\end{array}\right.
$$

With (6.18)-(6.20), it is a routine process to verify that $(a, u)$ satisfies the system (6.1) in the sense of distribution(see also [11]). Finally, following the argument in [11], we can show that $(a, u) \in C\left([0, T] ; \dot{B}_{p, 1}^{\frac{N}{p}}\right) \otimes C\left([0, T] ; \dot{B}_{p, 1}^{\frac{N}{p}-1}\right)$.

\section{The proof of uniqueness}

In this section, we prove the uniqueness of the solution. Assume that $\left(a^{1}, u^{1}\right) \in E_{T}^{p}$ and $\left(a^{2}, u^{2}\right) \in E_{T}^{p}$ are two solutions of the system (6.1) with the same initial data. Without loss of generality, we may assume that $a^{1}$ satisfies

$$
\rho^{1}(t, x)=\bar{\rho}_{0}\left(1+a^{1}(t, x)\right) \geq \frac{c_{0}}{2} .
$$

for any $(t, x) \in[0, T] \times \mathbb{R}^{N}$. Since $a^{2} \in C\left([0, T] ; \dot{B}_{p, 1}^{\frac{N}{p}}\right)$ and $\rho^{2}(0, x) \geq c_{0}$, there exists a positive time $\widetilde{T} \in(0, T]$ such that

$$
\rho^{2}(t, x)=\bar{\rho}_{0}\left(1+a^{2}(t, x)\right) \geq \frac{c_{0}}{2} .
$$

for any $(t, x) \in[0, \widetilde{T}] \times \mathbb{R}^{N}$. Set $\delta a=a^{1}-a^{2}$ and $\delta u=u^{1}-u^{2}$. Then $(\delta a, \delta u)$ satisfies

$$
\left\{\begin{array}{l}
\partial_{t} \delta a+u^{2} \cdot \nabla \delta a=\delta F-\delta u \cdot \nabla a^{1} \\
\partial_{t} \delta u-\operatorname{div}\left(\bar{\mu}^{1} \nabla \delta u\right)-\nabla\left(\left(\bar{\lambda}^{1}+\bar{\mu}^{1}\right) \operatorname{div} \delta u\right)=\delta G+\delta H \\
\left.(\delta a, \delta u)\right|_{t=0}=(0,0)
\end{array}\right.
$$

where

$$
\begin{aligned}
& \delta F=F\left(a^{1}, u^{1}\right)-F\left(a^{2}, u^{2}\right), \quad \delta G=G\left(a^{1}, u^{1}\right)-G\left(a^{2}, u^{2}\right), \\
& \delta H=\operatorname{div}\left(\left(\bar{\mu}^{1}-\bar{\mu}^{2}\right) \nabla u^{2}\right)+\nabla\left(\left(\bar{\lambda}^{1}-\bar{\lambda}^{2}+\bar{\mu}^{1}-\bar{\mu}^{2}\right) \operatorname{div} u^{2}\right),
\end{aligned}
$$

with $\bar{\lambda}^{i}=\bar{\lambda}\left(a^{i}\right), \bar{\mu}^{i}=\bar{\mu}\left(a^{i}\right)$ for $i=1,2$.

In what follows, we set

$$
U^{i}(t)=\int_{0}^{t}\left\|u^{i}(\tau)\right\|_{\dot{B}_{p, 1}^{\frac{N}{p}+1}} d \tau \quad \text { for } i=1,2,
$$

and denote by $A_{T}$ a constant depending on $\left\|a^{1}\right\|_{\widetilde{L}_{T}^{\infty}\left(\dot{B}_{p, 1}^{\left.\frac{N}{p}\right)}\right.}$ and $\left\|a^{2}\right\|_{\widetilde{L}_{T}^{\infty}\left(\dot{B}_{p, 1}^{\frac{N}{p}}\right)}$. 
Due to the inclusion relation $E_{T}^{p} \subseteq E_{T}^{N}$, it suffices to prove the uniqueness of the solution in $E_{T}^{N}$. So, we take $p=N$ in the sequel.

We apply Proposition 4.1 to get for any $t \in[0, T]$,

$$
\|\delta a(t)\|_{\dot{B}_{p, \infty}^{0}} \leq e^{C U^{2}(t)} \int_{0}^{t}\left(\|\delta F(\tau)\|_{\dot{B}_{p, \infty}^{0}}+\left\|\delta u \cdot \nabla a^{1}(\tau)\right\|_{\dot{B}_{p, \infty}^{0}}\right) d \tau .
$$

By Lemma 2.7, we have

$$
\begin{aligned}
\|\delta F(\tau)\|_{\dot{B}_{p, \infty}^{0}}+ & \left\|\delta u \cdot \nabla a^{1}(\tau)\right\|_{\dot{B}_{p, \infty}^{0}} \leq \\
& \leq C\left\|u^{2}\right\|_{\dot{B}_{p, 1}^{2}}\|\delta a\|_{\dot{B}_{p, \infty}^{0}}+C\left(1+\left\|a^{1}\right\|_{\dot{B}_{p, 1}^{1}}\right)\|\delta u\|_{\dot{B}_{p, 1}^{1}} .
\end{aligned}
$$

Plugging it into (7.2), we get by Gronwall's inequality that

$$
\|\delta a(t)\|_{\dot{B}_{p, \infty}^{0}} \leq e^{C U^{2}(t)} \int_{0}^{t}\left(1+\left\|a^{1}\right\|_{\dot{B}_{p, 1}^{1}}\right)\|\delta u\|_{\dot{B}_{p, 1}^{1}} d \tau .
$$

We use Proposition 5.3 to get for any $t \in[0, T]$,

$$
\begin{aligned}
\|\delta u(t)\|_{\widetilde{L}_{t}^{1}\left(\dot{B}_{p, \infty}^{1}\right)}+ & \|\delta u(t)\|_{\widetilde{L}_{t}^{2}\left(\dot{B}_{p, \infty}^{0}\right)} \leq \\
\leq & C \int_{0}^{t}\left(\|\delta G(\tau)\|_{\dot{B}_{p, \infty}^{-1}(\omega)}+\|\delta H(\tau)\|_{\dot{B}_{p, \infty}^{-1}}\right) d \tau \\
& +C A_{T}\left\|a^{1}\right\|_{\widetilde{L}_{t}^{\infty}\left(\dot{B}_{p, 1}^{1}(\omega)\right)}\|\delta u\|_{\widetilde{L}_{t}^{1}\left(\dot{B}_{p, \infty}^{1}\right)} .
\end{aligned}
$$

From Lemma 2.7, Proposition 3.7 and 3.8, we infer that for any $t \in[0, \widetilde{T}]$,

$$
\begin{aligned}
\|\delta H\|_{\dot{B}_{p, \infty}^{-1}} \leq & A_{T}\left\|u^{2}\right\|_{\dot{B}_{p, 1}^{2}}\|\delta a\|_{\dot{B}_{p, \infty}^{0}}, \\
\|\delta G\|_{\dot{B}_{p, \infty}^{-1}(\omega)} \leq & C\left\|\left(u^{1}, u^{2}\right)\right\|_{\dot{B}_{p, 1}^{1}}\|\delta u\|_{\dot{B}_{p, \infty}^{0}}+A_{T}\left\|a^{1}\right\|_{\dot{B}_{p, 1}^{1}(\omega)}\|\delta u\|_{\dot{B}_{p, \infty}^{1}} \\
& +A_{T}\left(1+\left\|u^{2}\right\|_{\dot{B}_{p, 1}^{2}}\right)\|\delta a\|_{\dot{B}_{p, \infty}^{0}} .
\end{aligned}
$$

We take $\widetilde{T}$ small enough such that

$$
\left\|\left(u^{1}, u^{2}\right)\right\|_{\widetilde{L}_{t}^{1}\left(\dot{B}_{p, 1}^{2}\right) \cap \widetilde{L}_{t}^{2}\left(\dot{B}_{p, 1}^{1}\right)}+\left\|\left(a^{1}, a^{2}\right)\right\|_{\widetilde{L}_{t}^{\infty}\left(\dot{B}_{p, 1}^{1}(\omega)\right)} \ll 1 .
$$

Thus, plugging (7.5) and (7.6) into (7.4), we infer that for any $t \in[0, \widetilde{T}]$,

$$
\|\delta u\|_{\widetilde{L}_{t}^{1}\left(\dot{B}_{p, \infty}^{1}\right)} \leq A_{T} \int_{0}^{t}\left(1+\left\|\left(u^{1}, u^{2}\right)\right\|_{\dot{B}_{p, 1}^{2}}\right)\|\delta a\|_{\dot{B}_{p, \infty}^{0}} d \tau .
$$

Lemma 7.1 ([13]) Let $s \in \mathbb{R}$. Then for any $1 \leq p, \rho \leq+\infty$ and $0<\epsilon \leq 1$, we have

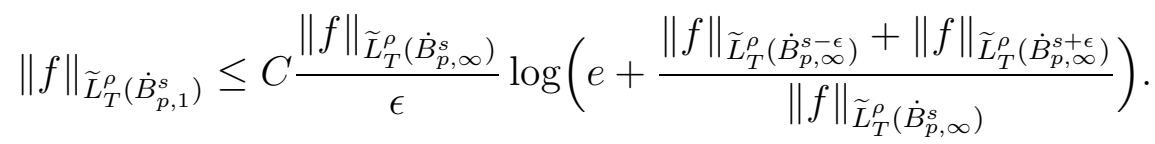


From Lemma 7.1, it follows that

$$
\|\delta u\|_{L_{t}^{1}\left(\dot{B}_{p, 1}^{1}\right)} \leq C\|\delta u\|_{\widetilde{L}_{t}^{1}\left(\dot{B}_{p, \infty}^{1}\right)} \log \left(e+\frac{\|\delta u\|_{\widetilde{L}_{t}^{1}\left(\dot{B}_{p, \infty}^{0}\right)}+\|\delta u\|_{\widetilde{L}_{t}^{1}\left(\dot{B}_{p, \infty}^{2}\right)}}{\|\delta u\|_{\widetilde{L}_{t}^{1}\left(\dot{B}_{p, \infty}^{1}\right)}}\right),
$$

which together with (7.3) and (7.7) yields that for any $t \in[0, \widetilde{T}]$,

$$
\|\delta u\|_{\widetilde{L}_{t}^{1}\left(\dot{B}_{p, \infty}^{1}\right)} \leq A_{T} \int_{0}^{t}\left(1+\left\|\left(u^{1}, u^{2}\right)\right\|_{\dot{B}_{p, 1}^{2}}\right)\|\delta u\|_{\widetilde{L}_{\tau}^{1}\left(\dot{B}_{p, \infty}^{1}\right)} \log \left(e+C_{T}\|\delta u\|_{\widetilde{L}_{\tau}^{1}\left(\dot{B}_{p, \infty}^{1}\right)}^{-1}\right) d \tau
$$

where $C_{T}=\|\delta u\|_{\widetilde{L}_{T}^{1}\left(\dot{B}_{p, \infty}^{0}\right)}+\|\delta u\|_{\widetilde{L}_{T}^{1}\left(\dot{B}_{p, \infty}^{2}\right)}$. Notice that $1+\left\|\left(u^{1}, u^{2}\right)(t)\right\|_{\dot{B}_{p, 1}^{2}}$ is integrable on $[0, T]$, and

$$
\int_{0}^{1} \frac{d r}{r \log \left(e+C_{T} r^{-1}\right)} d r=+\infty
$$

Osgood lemma applied concludes that $(\delta a, \delta u)=0$ on $[0, \widetilde{T}]$, and a continuity argument ensures that $\left(a^{1}, u^{1}\right)=\left(a^{2}, u^{2}\right)$ on $[0, T]$.

\section{References}

[1] Bony, J.-M.: Calcul symbolique et propagation des singularitiés pour les équations aux dérivées partielles non linéaires. Ann. Sci. École Norm. Sup. (4) 14 (1981), no. 2, 209-246.

[2] Bresch, D. And Desjardins, B.: Existence of global weak solutions for a 2D viscous shallow water equations and convergence to the quasigeostrophic model. Comm. Math. Phys. 238 (2003), no. 1-2, 211-223.

[3] Bresch, D., Desjardins, B. And Chi-Kun, L.: On some compressible fluid models: Korteweg, lubrication, and shallow water systems. Comm. Partial Differential Equations 28 (2003), no. 3-4, 843-868.

[4] Bresch, D., Desjardins, B. And Métivier, G.: Recent mathematical results and open problems about shallow water equations. In Analysis and simulation of fluid dynamics, 15-31. Adv. Math. Fluid Mech. Birkhäuser, Basel, 2007.

[5] Cannone, M.: Ondelettes, paraproduits et Navier-Stokes. Nouveaux essais. Diderot Editeur, Paris, 1995.

[6] Cannone, M.: Harmonic analysis tools for solving the incompressible Navier-Stokes equations. In Handbook of mathematical fluid dynamics. Vol. III, 161-244. North-Holland, Amsterdam, 2004.

[7] Chemin, J.-Y.: Perfect incompressible fluids. Oxford Lecture Series in Mathematics and its Applications 14. The Clarendon Press. Oxford University Press, New York, 1998. 
[8] Chemin, J.-Y.: Théorèmes d'unicité pour le système de Navier-Stokes tridimensionnel. J. Anal. Math. 77 (1999), 27-50.

[9] Chemin, J.-Y. And Lerner, N.: Flot de champs de vecteurs non lipschitziens et équations de Navier-Stokes. J. Differential Equations 121 (1995), no. 2, 314-328.

[10] Chen, Q., Miao, C. And Zhang, Z.: On the well-posedness for the viscous shallow water equations. SIAM J. Math. Anal. 40 (2008), no. 2, 443-474.

[11] Danchin, R.: Global existence in critical spaces for compressible NavierStokes equations. Invent. Math. 141 (2000), no. 3, 579-614.

[12] Danchin, R.: Local theory in critical spaces for compressible viscous and heat-conductive gases. Comm. Partial Differential Equations 26 (2001), no. $7-8,1183-1233$.

[13] Danchin, R.: Density-dependent incompressible viscous fluids in critical spaces. Proc. Roy. Soc. Edinburgh Sect. A 133 (2003), no. 6, 1311-1334.

[14] Danchin, R.: On the uniqueness in critical spaces for compressible NavierStokes equations. Nonlinear Differential Equations Appl. 12 (2005), no. 1, 111-128.

[15] Danchin, R.: Well-posedness in critical spaces for barotropic viscous fluids with truly not constant density. Comm. Partial Differential Equations 32 (2007), no. 7-9, 1373-1397.

[16] Fujita, H. And Kato, T.: On the Navier-Stokes initial value problem. I. Arch. Rational Mech. Anal. 16 (1964), 269-315.

[17] Haspot, B.: Cauchy problem for viscous shallow water equations with a term of capillarity. To appear in Math. Models Methods Appl. Sci. (2010). Doi 10.1142/S0218202510004532.

[18] Kazhikhov, A. V. And Shelukhin, V. V.: Unique global solution with respect to time of initial-boundary value problems for one-dimensional equations of a viscous gas. J. Appl. Math. Mech. 41 (1977), no. 2, 273-282.

[19] Lions, P.-L.: Mathematical Topics in Fluid Mechanics. Vol. 2, Compressible models. Oxford Lecture Series in Mathematics and its Applications 10. Oxford Science Publications. The Clarendon Press, Oxford University Press, New York, 1998.

[20] Matsumura, A. And Nishida, T.: The initial value problem for the equations of motion of compressible viscous and heat-conductive fluids. Proc. Japan Acad. Ser. A Math. Sci. 55 (1979), no. 9, 337-342.

[21] Mellet, A. And Vasseur, A.: On the barotropic compressible NavierStokes equations. Comm. Partial Differential Equations 32 (2007), no. 1-3, 431-452.

[22] Meyer, Y.: Wavelets, paraproducts and Navier-Stokes equations. In Current developments in Mathematics, 1996 (Cambridge, MA), 105-212. Int. Press, Boston, MA, 1997. 
[23] NASH, J.: Le problème de Cauchy pour les équation différentielles d'un fluide général. Bull. Soc. Math. France 90 (1962), 487-497.

[24] Triebel, H.: Theory of function spaces. Monographs in Mathematics 78. Birkhäuser Verlag, Basel, 1983.

[25] Wang, W.-K. And Xu, C.-J.: The Cauchy problem for viscous shallow water equations. Rev. Mat. Iberoamericana 21 (2005), no. 1, 1-24.

Recibido: 3 de diciembre de 2008

Qionglei Chen Institute of Applied Physics and Computational Mathematics Beijing 100088, China chen_qionglei@iapcm.ac.cn

Changxing Miao Institute of Applied Physics and Computational Mathematics Beijing 100088, China miao_changxing@iapcm.ac.cn

Zhifei Zhang School of Mathematical Science

Peking University Beijing 100871, China zfzhang@math.pku.edu.cn

Q. Chen and C. Miao were partially supported by the NSF of China under grant No. 10931001, No. 10725102. Z. Zhang was partially supported by the NSF of China under grant No. 10990013. 